\title{
Anomalies from Non-Perturbative Standard Model Effects
}

\author{
Holger Bech Nielsen*i \\ Niels Bohr Institutet, Copenhagen \\ E-mail: hbech@nbi.dk
}

\section{Colin D. Froggatt}

Glasgow University

E-mail: colin.froggattegla.ac.uk

\begin{abstract}
We interpret anomalies/deviations from the Standard Model as being in fact due to nonperturbative effects, because the top-yukawa-coupling is after all so large that non-perturbative effects become important. Most of the anomalies found have the character of signaling violation of lepton universality (LUV).

There are four lepton universality violating anomalies known at present, which we shall fit with one overall scale parameter $K$ to order of magnitude accuracy. One can look at the picture that we have found - due to the rather strong top-yukawa-coupling - as a new sector of strongly interacting particles analogous to QCD inside the Standard Model!

In addition we treat what is presumably also an anomaly: the experimental value of the direct CPviolation parameter $\varepsilon^{\prime}$ or $\varepsilon^{\prime} / \varepsilon$ turns out to be a factor 2 or more larger than the Standard Model prediction. It can also be fitted well by our model. However if we include in our anomaly model such processes not involving leptons (but only quarks), it seems superficially that we obtain very large - and phenomenologically unacceptable - anomalies in $K^{0} \bar{K}^{0}$ etc. mixing. To rescue our model from this falsification, we think of the interacting quarks as provided with clouds (on $1 / 2$ $\mathrm{TeV}$ scale) of, in our picture, the strongly interacting Higgs and top-quark particles, and that too strongly interacting such clouds cannot penetrate into each other. Thereby the interactions and thus the resulting anomalies are damped. Even with such a crutch for our theory we only barely manage to avoid an overlarge anomaly in the indirect $\mathrm{CP}$-violation parameter $\varepsilon$. Our modified model also predicts an anomalous contribution to the $K_{L}-K_{S}$ mass difference of the same order of magnitude as the experimental value, which is consistent with the (statistically insignificant) deviation from the Standard Model lattice value.
\end{abstract}

Corfu Summer Institute 2018 "School and Workshops on Elementary Particle Physics and Gravity" (CORFU2018)

31 August - 28 September, 2018

Corfu, Greece

\footnotetext{
* Speaker.

${ }^{\dagger}$ A footnote may follow.
} 


\section{Introduction}

As we shall list in section 2 there are (about) 5 small, most barely statistically significant, deviations from the Standard Model. Interestingly enough 4 out of these 5 anomalies involve violation of lepton universality. That is to say, there is an anomaly for one lepton flavour rather than for another one, or at least the anomaly is different for e.g. electron and muon. Precisely such a muon versus electron difference is the point of "the $R(K)$ and $R\left(K^{*}\right)$ anomaly in B-decay", "the muon magnetic moment anomalous magnetic moment anomaly" (there is namely no anomaly in the electron anomalous magnetic moment), and "the proton radius puzzle", which consists in the fact that the radius of the proton is fitted to be smaller for muonic data than for the usual electronic atomic data. "The $R(D), R\left(D^{*}\right)$, and $R(J / \Psi)$ in B-decay anomaly" is a violation of the universality between the $\tau$-lepton and the lighter leptons, $\mu$ say. The only one of the treated anomalies, which is not about lepton universality violation, is "the $\varepsilon^{\prime} / \varepsilon$ anomaly" concerned with direct CP-violation.

It is our point to fit all these 5 anomalies as being due to non-pertubative effects present in the Standard Model - so that strictly speaking the Standard Model is perfectly o.k. - order of magnitudewise with only one overall scale parameter, which we call $K$.

We shall see below that the fitting of the very different observed anomalies works very well. But we have the problem, that we must "improve" on our model, to avoid it making predictions for outrageous anomalies in neutral meson mixings, $K^{0} \bar{K}^{0}$ and so on. Ideas for such an improvement are included below, but it might be easier to take a version of our model in which we only consider processes with a lepton. If we do so we strictly speaking sacrifice the "the $\varepsilon^{\prime} / \varepsilon$ anomaly in $\mathrm{CP}$ -violation", in spite of it in fact fitting very well using the overall scale parameter $K$-value obtained by fitting to the lepton involving anomalies.

The philosophical point of our fitting of the anomalies with the overall scale parameter $K$ is that the top-Yukawa coupling in the Standard Model $g_{t}=0.93_{5}$ is close to being of order unity and thus could in principle be suspected to - much like the strong gauge coupling in QCD $\alpha_{S}$, which runs large in the low energy regime - give rise to non-perturbative effects becoming important. In fact we propose that the top-Yukawa coupling $g_{t}$ is so big as to give rise to a "new strong interaction sector", in which then this top-Yukawa coupling plays an analogous role to the strong gauge coupling in QCD. Whether a coupling like $g_{t}$ should really be considered sufficiently strong to cause significant non-perturbative effects - such as strongly bound states, or significant effective interactions of a more complicated type - does not only depend on the coupling being sufficiently strong/large but also on how many different species of particles that couple to each other by means of this coupling. At the end we shall give some estimates actually ending up with the conclusion that the $g_{t}=.935$ is indeed almost sufficient to be expected to give severe non-perturbative effects.

In the next section 2 we shall list the deviations from the Standard Model found and review the main experimental results.

Then in the section 3 we put forward the rules for our model.

In section 4 we go through the various anomalies found and fit our parameter $K$, an overall scale for the non-perturbative effect.

In section 5 we present in a table the success of our model: we get almost the same value for our parameter $K$ by fitting whichever of the anomalies. 
In section 6 we briefly discuss what the "strong coupling", which is the basis for nonperturbative effects, actually stands for; first it means of course that we do not in the true sense have any new physics, so that the Standard Model can still be perfect. However it might also be considered a support for our long discussed multiple point principle, that there are several degenerate vacua. Thirdly we estimate whether the value $g_{t}=0.935$ is expected to be sufficiently strong to indeed give non-perturbative effects; we find that it is close to the border line for being so strong as to give significant non-perturbative effects.

In section 7 we worry about, how to avoid our model being totally falsified by predicting too large anomalies in the pseudoscalar meson mixings, such as $K^{0} \bar{K}^{0}$ mixing.

For this purpose we invent a mechanism, that may for very strongly interacting particles prevent them from penetrating into the clouds of top and Higgs particles around them and thus damp the predictions of our model. This penetration story is described in section 8 .

Section 9 is assigned to a discussion of the prediction of our modified model of an anomaly in the $\mathrm{CP}$-violating parameter $\varepsilon$ measured in the first $\mathrm{CP}$-violating experiment by Christensen, Cronin et al. Really this $\varepsilon$ parameter seems to fit well to the Standard Model. Although at first it looks that our predicted anomaly is dramatic, we explain in this section, that it is not that serious.

In section 10 we conclude with a résumé and give some PREdictions for where soon to find further anomalies.

\section{The Anomalies}

We think we should stress that the type of experiments in which one has found anomalies, in the sense of deviations from the Standard Model predictions, are of very different character: The first two anomalies though are both deviations from lepton universality (LU) in B-meson decays. But even these two a priori similar deviations differ in fact rather remarkably by the first one " $R(K)$ and $R\left(K^{*}\right)$ " corresponding to a process, that is of extremely low decay rate, because it is a neutral (weak) current process with a couple of charged leptons in the final state, while the next anomaly " $R\left(D^{*}\right)$ and $R(j / \Psi)$ " concerns a charged current process, thus with a much bigger decay rate. The muon and electron anomalous magnetic moments are measured with very special instrumentation and calculated theoretically with extreme accuracy. The "Proton radius puzzle" is really that atomic physics data with a muon instead of an electron is fitted by a radius for the proton $4 \%$ smaller than that obtained from the electronic/usual atomic data.

If we should choose to only consider the anomalies in processes with leptons - and in fact lepton universality violation - we obtain our reduced model. We should then ignore the $\varepsilon^{\prime} / \varepsilon$ anomaly, but really we also manage to fit that non-leptonic anomaly very well.

But the truth is that we have to produce a crutch - a story about the quarks being stopped on the way penetrating into each other or rather each others surrounding clouds of say top and Higgs particles - for our model for treating the non-perturbative effects, in order to avoid predicting too huge anomalies for the mixing of the neutral mesons such as $K^{0} \bar{K}^{0}$. Even after such improvement it is hard to keep the anomaly in especially the indirect CP-breaking parameter $\varepsilon$ sufficiently small. (We assign section 9 to this problem).

As the lepton family in our model comes in via a number of lepton-Higgs-yukawa couplings, we tend to get much bigger anomalies the bigger the mass of the lepton in question. Therefore 
we shall with our model in mind always think of the heaviest lepton involved, in the comparisons showing lepton universality violation, as being the lepton flavour carrying the anomaly. When as in most cases the lack of universality is between muon and electron, we shall therefore take it that it is the muon process that is anomalous. It is a confirmation of this point of view that the anomalous magnetic moment for the muon has an anomaly, while the electron anomalous magnetic moment fits the Standard Model perfectly. Only in the case number 2 below, "the $R(D), R\left(D^{*}\right)$, and $R(J / \Psi)$ anomaly in B-decay", it is not the muon but the $\tau$-lepton process that shall carry the anomaly (it is only in this case that the corresponding $\tau$-process has been sufficiently well measured.)

\subsection{The $R(K)$, and $R\left(K^{*}\right)$ anomaly in B-decay}

While the usually dominating weak decays are of so called charged current type, which have the highest rates, the first anomaly[1] called " $R(K)$ and $R\left(K^{*}\right)$ " concerns in fact processes in which a pair of oppositely charged leptons are produced, so that the lepton pair is neutral and thus must have coupled to a neutral current, if one thinks this way. On the figure [2] you see the diagrams in normal perturbative thinking for the two different types of processes, charged and neutral currents.

\section{B decays and Lepton Universality}
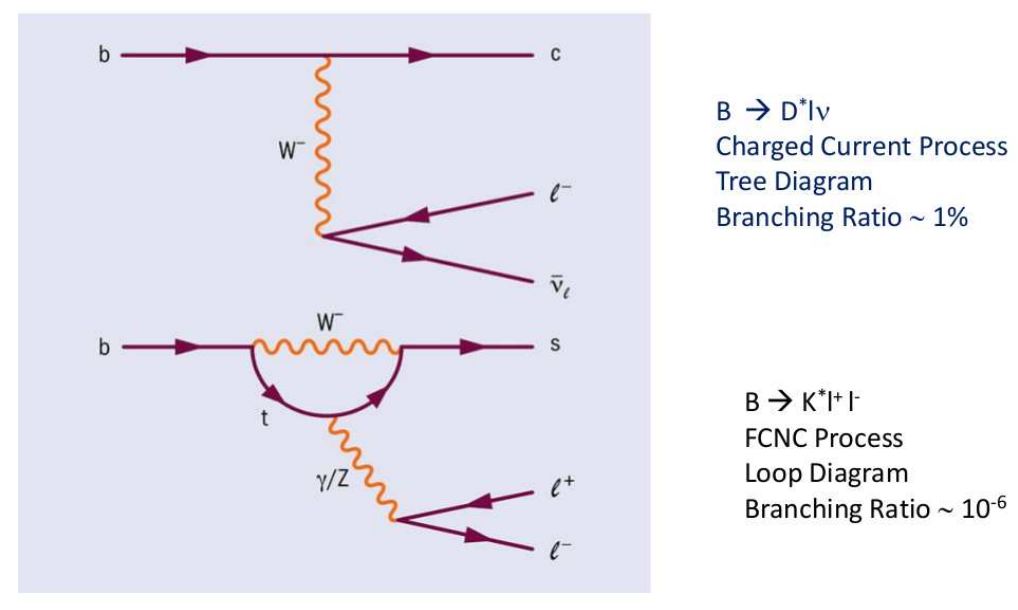

The ratios studied - and found to be too small compared to the Standard Model prediction, unity in this case - are the ratios of the decay rate of a B-meson to respectively a $K$ or a $K^{*}$ and in the numerator a $\mu^{+} \mu^{-}$pair while in the denominator instead an $e^{+} e^{-}$pair. One should consider the similarity of the values measured for these two ratios as increasing the statistics for there being any effect at all: 


\section{$\mathrm{B} \rightarrow \mathrm{K}^{*} \mathrm{I}^{+} \mathrm{I}^{-}$}

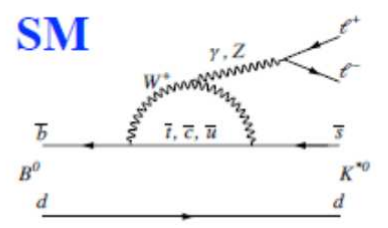

Penguin Diagram

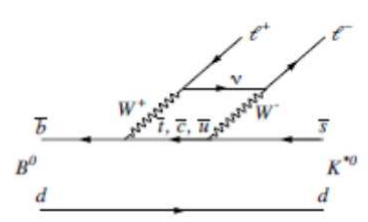

Box diagram

Uncertainties in SM predictions due to decay form factors cancelled by taking the ratio

$$
R\left(K^{*}\right)=\frac{B\left(B \rightarrow K^{*} \mu^{+} \mu^{-}\right)}{B\left(B \rightarrow K^{*} e^{+} e^{-}\right)}
$$

Lepton Universality $\Longrightarrow R\left(K^{*}\right)_{S M}=1$. Similarly $R(K)_{S M}=1$

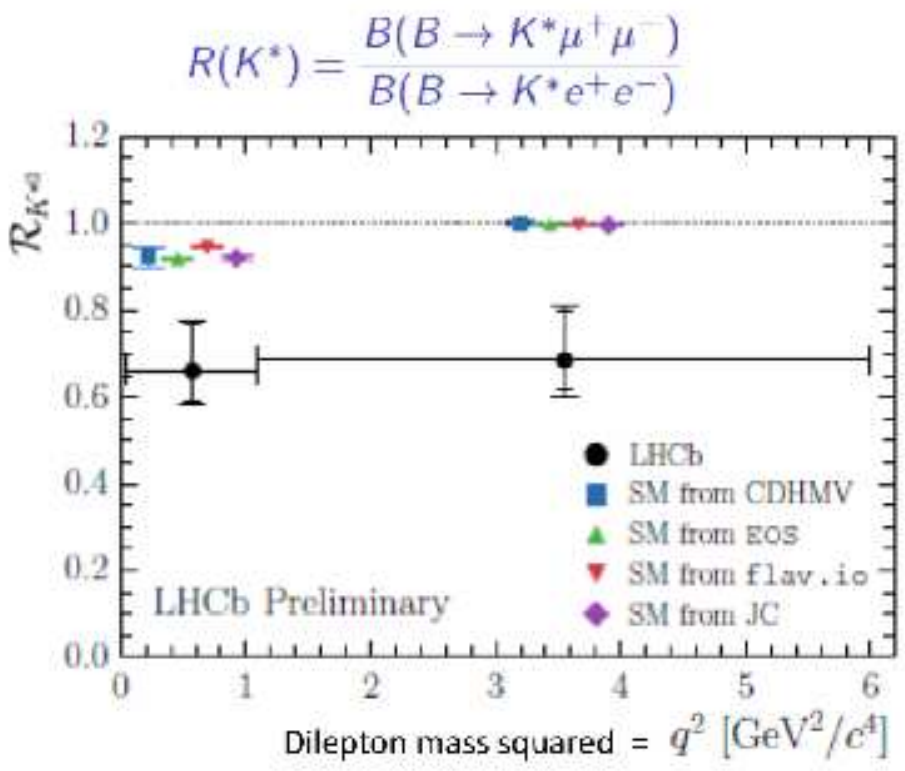




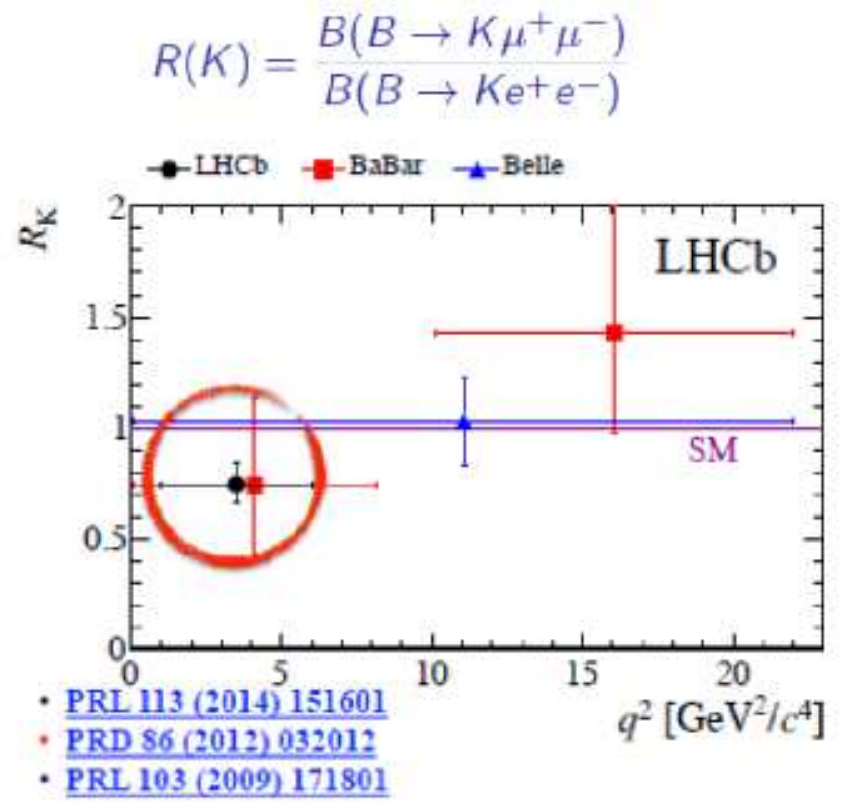

Lepton Universality Violation in $\mathrm{B} \rightarrow \mathrm{KI}^{+} \mathrm{I}^{-}$and $\mathrm{B} \rightarrow \mathrm{K}^{*} \mathrm{I}^{+} \mathrm{I}^{-}$

$$
\begin{aligned}
& R_{K}=0.745^{+0.09}{ }_{-07} \text { (stat) } \pm 0.036 \text { (syst) } \\
& R_{K_{C}}=0.66^{+0.11}-0.07 \text { (stat) } \pm 0.03 \text { (syst) } 0.045<q^{2}<1.1 \mathrm{GeV}^{2} \\
& R_{K}=0.69^{+0.11}-0.07 \text { (stat) } \pm 0.05 \text { (syst) } \quad 1.1<q^{2}<6 \mathrm{GeV}^{2}
\end{aligned}
$$

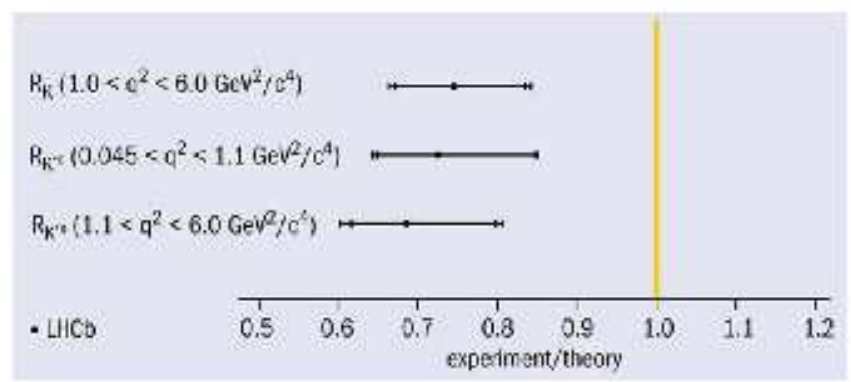

\subsection{The $R(D), R\left(D^{*}\right)$, and $R(J / \Psi)$ anomaly in B-decay}

An anomaly[3] has been found in the ratio

$$
R\left(D^{(*)}\right)=\frac{\Gamma\left(B \rightarrow D^{(*)} \tau v_{\tau}\right)}{\Gamma\left(B \rightarrow D^{(*)} \mu v_{\mu}\right)}
$$

(and also for the corresponding ratio $R(D)$ ), which if different from its Standard Model value would represent a lack of lepton universality between $\tau$ and $\mu$. Measurements of decays involving electrons and muons show no deviations with respect to the Standard Model within the current level of precision. 
The Standard Model values for $R\left(D^{*}\right)$ and $R(D)$ differ from unity due to the phase space difference between the $\tau$ and $\mu$ processes:

$$
R(D *)_{S M}=0.252 \pm 0.003 \quad \text { and } \quad R(D)_{S M}=0.299 \pm 0.006
$$

\section{Summary of $R(D), R\left(D^{*}\right)$ and $R(J / \psi)$}
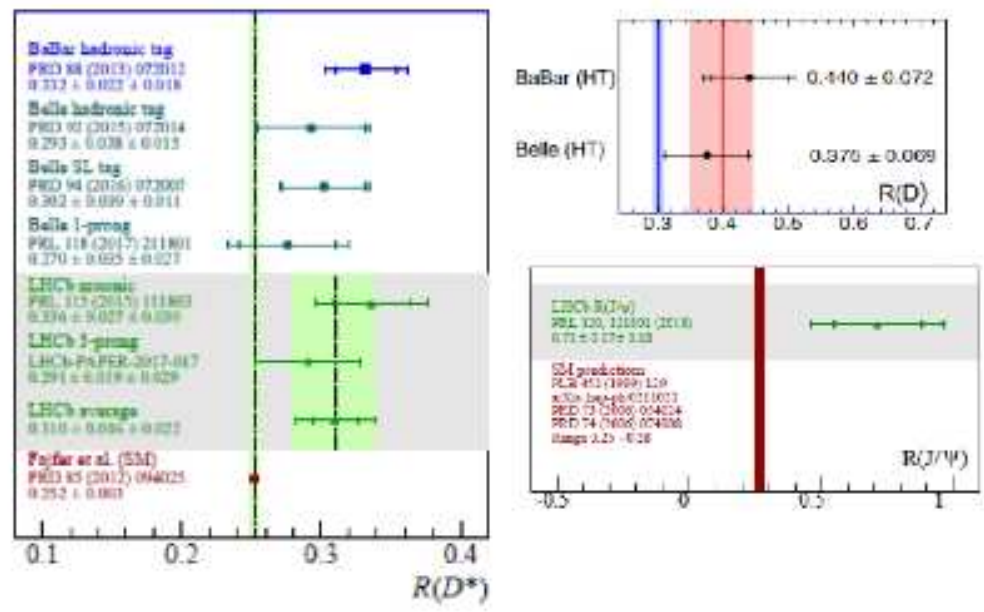

\subsection{The $\mu$ anomalous magnetic moment anomaly}

The anomalous magnetic moments for both muon and electron have been measured with high accuracy and compared to the theory; only the one for the muon deviates slightly from the Standard Model [4].

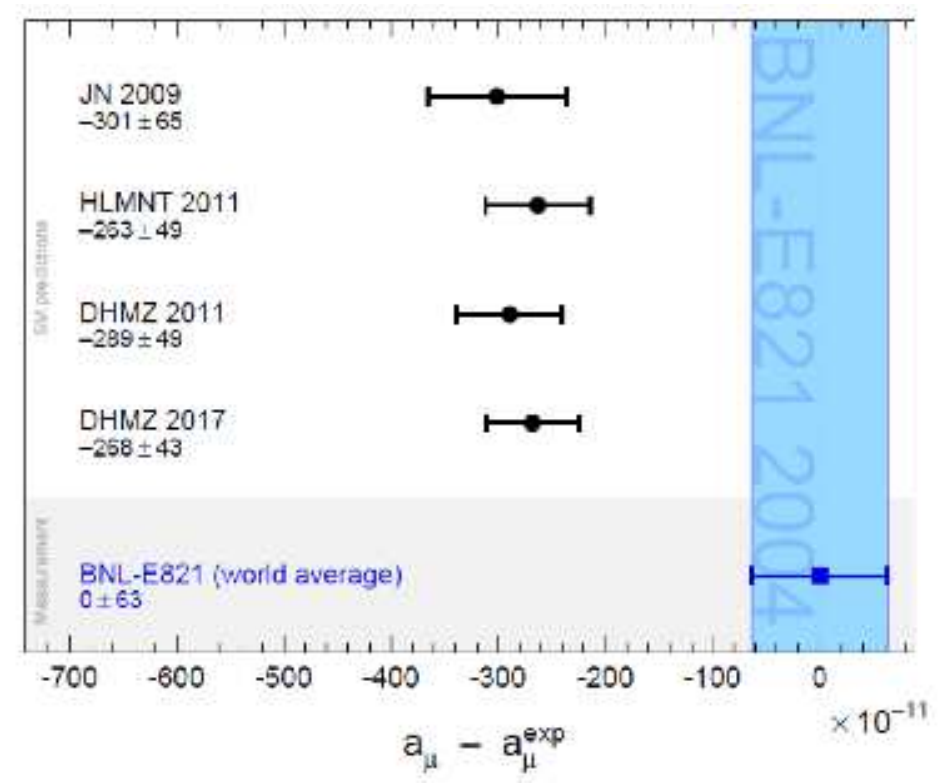




\section{Muon Anomalous Magnetic Moment}
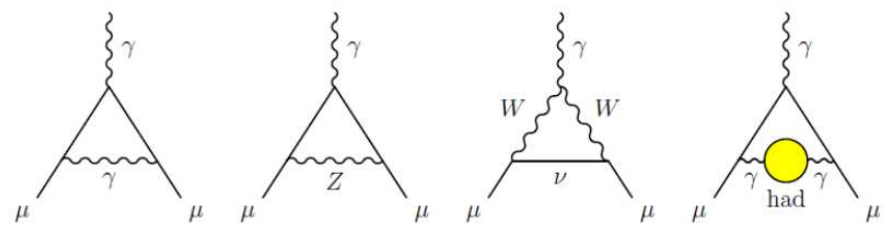

Lowest order QED, Electroweak and Hadronic Diagrams

$$
\begin{aligned}
& a_{\mu}^{\mathrm{SM}}=116591823(1)(34)(26) \times 10^{-11} \\
& a_{\mu}^{\exp }=11659209.1(5.4)(3.3) \times 10^{-10} \\
& \Delta a_{\mu}=a_{\mu}^{\exp }-a_{\mu}^{\mathrm{SM}}=268(63)(43) \times 10^{-11}
\end{aligned}
$$

\subsection{Proton Radius Puzzle}

In 2010 Antognini et al. [5, 6] measured the muonic hydrogen atom spectrum so as to find what is known as the "Proton Radius Puzzle", namely that the radius of the proton fitted to the muonic atom spectroscopy turns out $4 \%$ or $5.6 \sigma$ smaller than the radius measured in electronic atoms and e-p scattering. A similar effect is seen in deuterium [7]. Using respectively electrons and muons in the atom one gets

$$
\begin{aligned}
R_{p} \text { using electrons } & =0.8768 \pm 0.0069 \mathrm{fm} \\
R_{p \text { using muon in the atom }} & =0.842 \pm 0.001 \mathrm{fm} \text { deviating by } 5 \text { s.d. }
\end{aligned}
$$

An overview-talk [8].

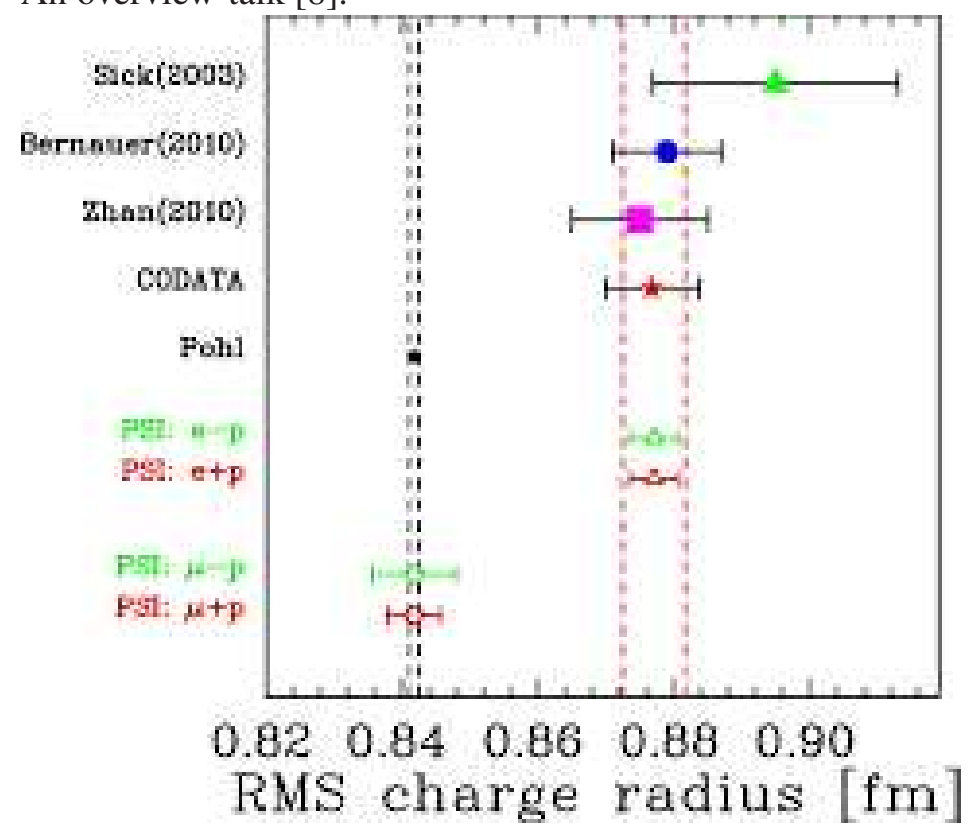




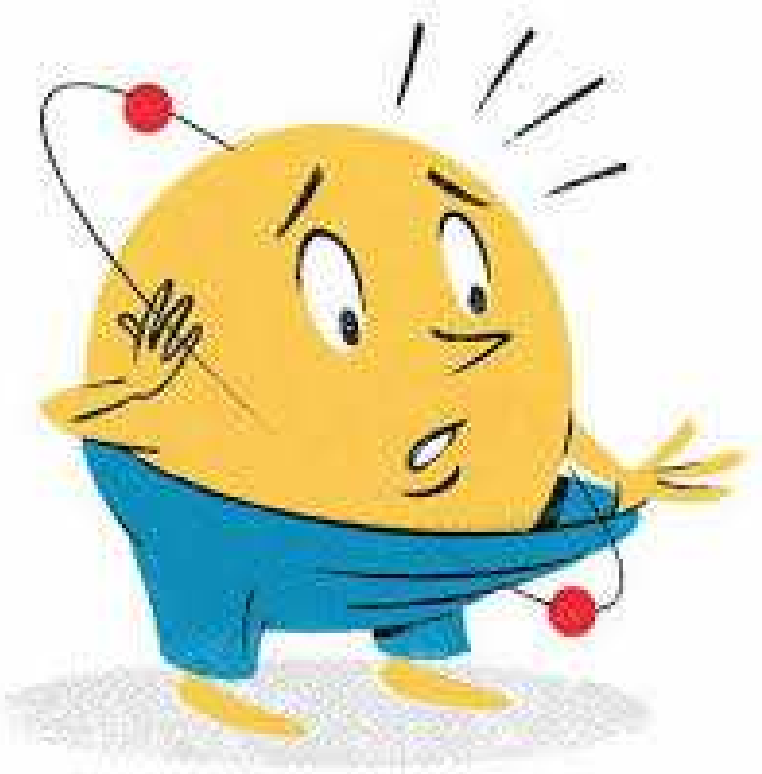

2.5 The $\varepsilon^{\prime} / \varepsilon$ anomaly in $K \bar{K}$-mixing.

Indirect versus direct $\mathrm{CP}$ violation in $K_{L} \rightarrow \pi \pi$

$$
\begin{aligned}
& K_{1}=\frac{1}{\sqrt{2}}\left(K^{0}-\bar{K}^{0}\right), \quad C P\left|K_{1}\right\rangle=\left|K_{1}\right\rangle \rightarrow 2 \pi \\
& K_{2}=\frac{1}{\sqrt{2}}\left(K^{0}+\bar{K}^{0}\right), \quad C P\left|K_{2}\right\rangle=-\left|K_{2}\right\rangle \rightarrow 3 \pi \\
& K_{\mathrm{S}}=\frac{K_{1}+\bar{\varepsilon} K_{2}}{\sqrt{1+|\bar{\varepsilon}|^{2}}}, \quad K_{\mathrm{L}}=\frac{K_{2}+\bar{\varepsilon} K_{1}}{\sqrt{1+|\bar{\varepsilon}|^{2}}} \\
& K_{L} \propto K_{2}+\bar{\varepsilon} K_{1} \\
& \underset{\text { indirect }: \varepsilon}{\text { direct }: \varepsilon^{\prime}} \longrightarrow \pi \pi
\end{aligned}
$$


Box diagrams for indirect $\mathrm{CP}$ violation
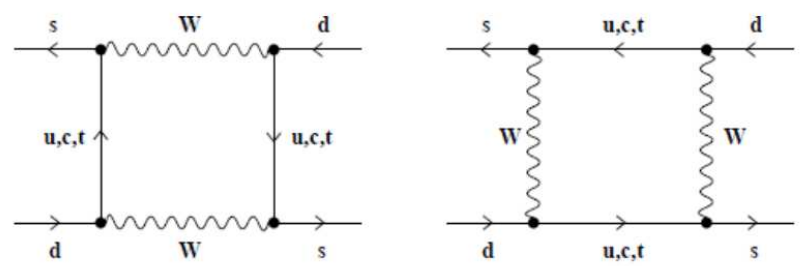

Penguin diagrams for direct $\mathrm{CP}$ violation
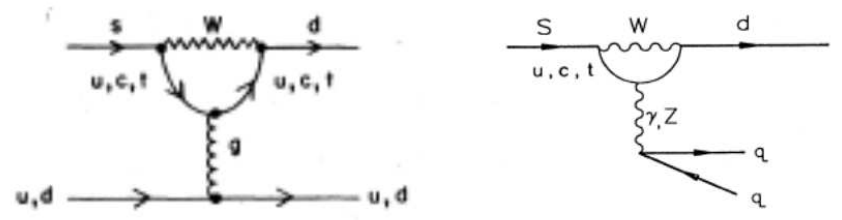

Penguin Diagram

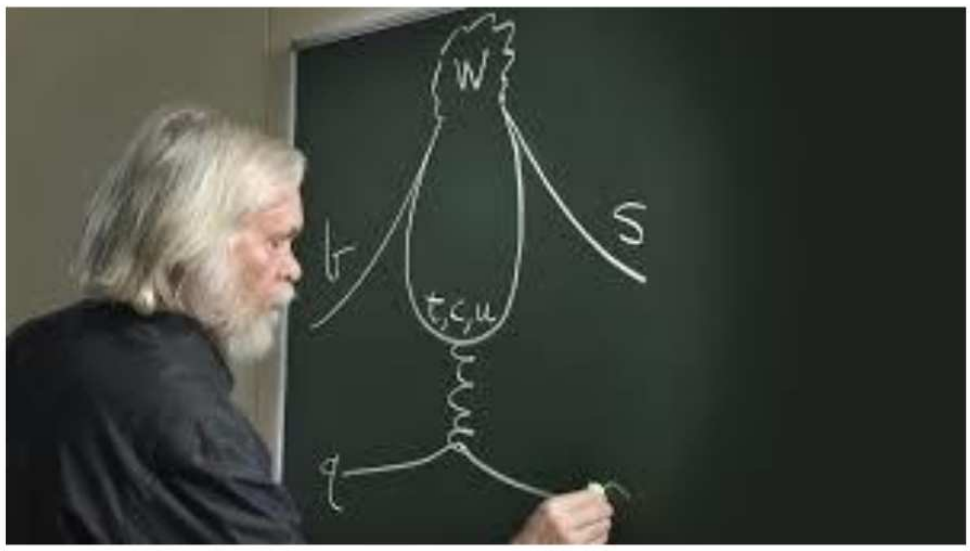




\section{CP Violation Parameters}

$$
\begin{aligned}
& \text { Indirect CP } \quad|\epsilon|=(2.228 \pm 0.011) \times 10^{-3} \\
& \text { Fitted by CKM parameters in SM } \\
& \begin{aligned}
\text { Direct CP } \quad\left(\varepsilon^{\prime} / \varepsilon\right)_{\exp }=(16.6 \pm 2.3) \times 10^{-4} \\
\left(\varepsilon^{\prime} / \varepsilon\right)_{\mathrm{SM}}=(1.38 \pm 6.9) \times 10^{-4}
\end{aligned} \\
& \text { So }\left(\frac{\varepsilon^{\prime}}{\varepsilon}\right) \text { dominated by anomalous term and } \\
& \epsilon_{\exp }^{\prime}=2.228(16.6 \pm 2.3) * 10^{-7}=(3.6 \pm 0.5) * 10^{-6}
\end{aligned}
$$

The direct CP-violation in $\mathrm{K} \rightarrow>2$ pions $\varepsilon^{\prime}$ is hard to calculate in the Standard Model but gives a small value compared to the experimental one $[9,10]$ and thus the anomaly should even dominate.

\section{Our non-perturbative model}

The philosophy of our model or interpretation of the anomalies is that there is "a new strong sector" in addition to QCD. That is to say, the Standard Model is actually completely correct as the fundamental model; however, due to the appearance of the top-quark Yukawa coupling $g_{t}=0.935$ which is of order unity, there can and actually we suppose do appear non-perturbative effects. We shall return below in section 6 to the question as to what is the borderline for a coupling constant so as to go from being one for which perturbation theory as usual works to one for which nonperturbative effects are to be expected. We find that, with say about 16 different particle or better components (including color and spin variants), the borderline coupling between strong and weak values for the top Yukawa coupling is

$$
g_{t \text { border }} \approx(6 \text { to } 4 \pi) / \sqrt{16}=1.5 \text { to } 3 .
$$

The idea is that new phenomena not found in pure perturbation theory, such as bound states or new condensates, will show up because of the strong coupling. We, however, seek to not commit ourselves into details as to what sort of new phenomena should pop up, but rather think so abstractly about it that we only think about very high order Feynman diagrams being important. We can then hope our estimations are o.k. almost independently of precisely which type of non-perturbative phenomenon pops up, bound states or new phases,...

Indeed we assume, that the new feature in our model is some very complicated very high order diagrams which, except for some smaller attachments to the studied particles, consist only of vertices of the top-Yukawa coupling type. Then of course these vertices must be connected by propagators for particles coupling by means of this Yukawa coupling. That is to say that in the bulk of the diagram we find only the top quarks - right and left chirality top propagators - and the Higgs particle propagator. 


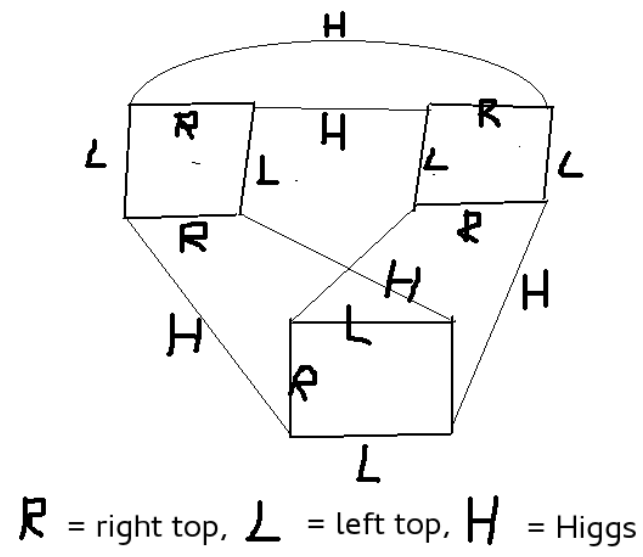

On the figure we see a vacuum diagram with only these particle and only the $g_{t}$-vertex.

\section{Non-Perturbative Model}

\section{Large Top-Higgs Yukawa coupling $g_{t} \sim 1$.}

Our suggestion:

- Complicated higher order diagrams involving top and Higgs particles generate so far ignored non-perturbative effects.

- Sum over these diagrams with only $g_{t}$ couplings gives one overall factor $K$, which we fit to data.

- Attach a few external lines of other types to generate dim $=6$ effective terms responsible for the experimental anomalies.

- Momenta inside diagram typically $\sim 1 \mathrm{TeV}$.

- So can neglect masses in propagators and obtain formal weak $\mathrm{SU}(2)$ conservation in diagrams.

\subsection{More precise rule}

In practice we let our diagrams with a lot of $g_{t}$-vertices and only a few different mainly external particles be arranged to simulate a dimension $=6$ effective field theory Lagrangian density term. This is of course a reflection of the assumption that the whole non-perturbative effect is short range compared to the scale of energy at which the anomalies are observed. In fact we have in mind an energy scale of the order of $1 / 2 \mathrm{TeV}$, say.

To make precise sense of our overall weight factor $K$ we have to make some rule even for the normalization of the dimension 6 field combinations supposed to come out of our non-perturbative effect. Using this normalization we can then say that the coefficient shall just be $K$ modified by being multiplied by various suppression factors. The rule of normalization, which we propose to use here, is that the dimension 6 operator - to be considered properly normalized - shall be the 
product of two dimension 3 operators each of which is normalized in analogy to the expressions used to normalize the wave functions for the particles in the following way:

Let us first remind ourselves about the way one usually normalize wave functions:

- Spin $1 / 2$ fermion

$$
\begin{aligned}
<\psi_{1} \mid \psi_{2}> & =\int \bar{\psi}_{1}(x) \gamma^{0} \psi_{2}(x) d^{3} x \\
& =\int \psi_{1}^{\dagger}(x) \psi_{2}(x) d^{3} x
\end{aligned}
$$

or equally good for positive energy single particle states

$$
<\psi_{1} \mid \psi_{2}>=\int \bar{\psi}_{1}(x) \psi_{2}(x) d^{3} x
$$

- Bosons

For Bosons with complex (non-hermitean) fields:

$$
<\phi_{1} \mid \phi_{2}>=\int \phi_{1}^{\dagger}(x) \stackrel{\leftrightarrow}{\partial_{0}} \phi_{2}(x) d^{3} x
$$

while for real (hermitean) fields we have a factor 2 less:

$$
<\phi_{1} \mid \phi_{2}>=\int \phi_{1}^{\dagger}(x) \partial_{0} \phi_{2}(x) d^{3} x
$$

The reason for this difference between the "complex" and the "real" cases, may be seen by first noticing, that if one wants both for real and complex fields to have a propagator of the form

$$
\operatorname{prop}\left(p^{\mu}\right)=\frac{i}{p^{2}-m^{2}}
$$

without any extra factor 2 depending on the reality, then it is needed to take say the kinetic term in the Lagrangian density or the Hamiltonian density to have a factor $1 / 2$ extra in the real case:

$$
\begin{aligned}
& \mathscr{L}(x)=\partial_{\rho} \phi^{*} \partial^{\rho} \phi+\ldots \text { for complex field }, \\
& \mathscr{L}(x)=\frac{1}{2} \partial_{\rho} \phi \partial^{\rho} \phi+\ldots \text { for real field. }
\end{aligned}
$$

The point is that when you derive the propagator from the action by functional differentiation you cannot in the real case avoid obtaining the factor 2 from differentiating a square, while in the complex case one can formally play as if $\phi$ and $\phi^{*}$ were independent variables and avoid the factor 2 .

In fact we shall meet below, in our discussion of the anomalous magnetic moment of the muon, a mixed case of "complex" with a "real" and propose to use as compromise a square root 2 factor, $\sqrt{2}$. 
The idea then is that we construct the dimension 6 field combination by inserting in these expressions the second quantized field, $\psi \rightarrow \boldsymbol{\psi}$ and $\phi \rightarrow \boldsymbol{\phi}$, and multiply two of these normalized expressions together.

Next we make this a priori not relativistic expression of dimension 6 covariant, by supplementing it with components of the same four vectors so that we get ordinary four vector contractions. We should really even consider tensor or scalar contractions between the two "currents" which are w.r.t. the 0-indices the term going into the number of particles normalization. (In fact we shall see that we shall use a tensor contraction for the case of the anomaly in the anomalous magnetic moment for the muon.)

For example: After having made a product say

$$
\overline{\boldsymbol{\psi}}_{1}(x) \gamma^{0} \boldsymbol{\psi}_{2}(x) * \overline{\boldsymbol{\Psi}}_{3}(x) \boldsymbol{\gamma}^{0} \boldsymbol{\psi}_{4}(x)
$$

we make it covariant as follows

$$
\overline{\boldsymbol{\psi}}_{1}(x) \boldsymbol{\gamma}^{\mu} \boldsymbol{\psi}_{2}(x) * \overline{\boldsymbol{\psi}}_{3}(x) \gamma_{\mu} \boldsymbol{\psi}_{4}(x)
$$

which is then our "normalized" dimension 6 effective term. It should be stressed, that we must make a normalization condition like this one in order to ever be able to have our model give more than order of magnitude results. However, since we anyway shall supplement by rules of inserting a factor $g_{l} / g_{t}$ for each lepton 1 needed Yukawa coupling, we can hardly hope for more than an order of magnitude accuracy anyway; but without the normalization specification, we would even in principle be excluded from having a better accuracy than order of magnitude at most. At the end we find somewhat surprisingly, that the agreement is so good, that it is almost better than order of magnitude; then this is not a priori without meaning as long as we use this normalization rule, but surprising anyway.

You can extract external particles without any extra - and thus lower strength - vertices when they are the ones already participating in the big complicated diagram. That is to say you can extract:

- Higgs particles both the "radial one" (the experimentally found (radial) Higgs boson) and the "eaten ones" meaning truly longitudinal W's and Z's.

- Genuine top-quarks

- The left-handed dsb-quark combination partner of the top-quark. This means a superposition of three left chirality components of the quarks down, strange, and bottom, which is just that combination, which is in the weak isospin doublet with the left chirality top quark. These particles namely also couple via the strong top Yukawa coupling constant $g_{t}$.

If we want to have other external particles in the effective coupling to be identified as part of the non-perturbative effects, then at least one or more extra propagators have to be added to the complicated diagram. If the extra propagator - say a $W$ as we need in the "The $R(D), R\left(D^{*}\right)$, and $R(J / \Psi)$ anomaly in B-decay" case - is considered to sit well inside the complicated diagram, so that the loop momenta passing through it are of the typical scale of the non-perturbative diagram, $\sim 1 / 2$ 
$\mathrm{TeV}$, we may effectively ignore the propagator. Then we only correct our effect by a factor coming from the deviation of the attachment couplings of the extra propagator from the $g_{t}$-coupling.

The point that the "light quarks"(meaning all but the top quark) couple only immediately as left handed chirality particles means, that - at least without severe extra propagators added - the most important effective field term coming out of the model will have its quark part solely involving left chirality dsb-quarks (i.e. no quarks with $2 / 3$ charge except the top itself). Put as a current for the quarks we thus have a left handed projector $P_{L}$ inserted.

For the lepton coupling we have a priori both handednesses possible. However, concerning the lepton, we still have to note that it can only couple to the Higgs among the particles strongly interacting via the $g_{t}$ coupling, in as far as the leptons do not couple directly to the top quark.

Now we must further notice that on the short distance scale, large energy scale $1 / 2 \mathrm{TeV}$, the vacuum Higgs field is small and is only expected to come in, if absolutely needed. This is the case for the magnetic moment anomaly, which is an anomaly in a term needing its proportionality to the Higgs field expectation value $\langle\boldsymbol{\phi}\rangle$ because the anomalous magnetic moment coupling of the muon corresponds to a left to right transition or opposite.

We indeed have conservation of the weak isospin in our complicated diagrams. So, if an even number of the effective left chirality quarks come out of the diagram, the number of Higgses coming out must be even too. The term we can get with say $\overline{\boldsymbol{s}} \gamma^{v} P_{L} \boldsymbol{b}=\overline{\boldsymbol{\psi}}_{s} \gamma^{v} P_{L} \boldsymbol{\psi}_{b}$ as the one factor in fitting the $R\left(K^{*}\right)$ anomaly can have either only left or only right say muons in the other factor, and thus be like $\overline{\boldsymbol{\mu}} \gamma_{\nu} \boldsymbol{\mu}=\overline{\boldsymbol{\psi}}_{\mu} \gamma_{\nu} \boldsymbol{\psi}_{\mu}$, or such a factor with a $\gamma^{5}$ inserted. We are in this way guided towards the operators $O_{9}$ or $O_{10}$ in the effective Hamiltonian (see below) and not even the primed operators, which would have signaled right handed chirality for the quarks. Our model so to speak predicts the unprimed $O_{9}$ or $O_{10}$ operators rather than the $O_{9}^{\prime}$ or $O_{10}^{\prime}$ operators to have the anomaly.

\section{Fitting $K$}

\subsection{Fitting $K$ for $R\left(K^{(*)}\right)$ anomaly}

Indeed by fitting the effective term needed, using a single operator in addition to the pure Standard Model, it has been found [11] that a value $C_{9}=-1.3$ for the coefficient to the operator $\mathrm{O}_{9}$ is needed. 


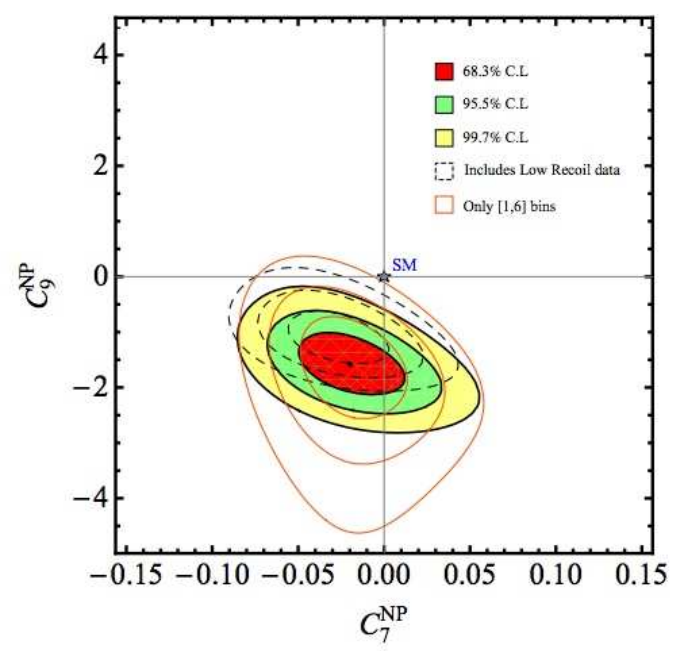

FIG. 1: Fit to $\left(\mathcal{C}_{7}^{\mathrm{NP}}, \mathcal{C}_{9}^{\mathrm{NP}}\right)$, using the three large-recoil bins for $B \rightarrow K^{*} \mu^{+} \mu^{-}$observables, together with $B \rightarrow X_{s} \gamma, B \rightarrow$ $X_{s} \mu^{+} \mu^{-}, B \rightarrow K^{*} \gamma$ and $B_{s} \rightarrow \mu^{+} \mu^{-}$. The dashed contours include both large- and low-recoil bins, whereas the orange (solid) ones use only the 1-6 $\mathrm{GeV}^{2}$ bin for $B \rightarrow K^{*} \mu^{+} \mu^{-}$ observables. The origin $\mathcal{C}_{7,9}^{\mathrm{NP}}=(0,0)$ corresponds to the $\mathrm{SM}$ values for the Wilson coefficients $\mathcal{C}_{7 \mathrm{eff}, 9}^{\mathrm{SM}}=(-0.29,4.07)$ at $\mu_{b}=4.8 \mathrm{GeV}$.

We use this fitting to estimate the value of our overall coefficient $K$ from the " $R(K)$ and $R\left(K^{*}\right)$ anomaly in B-decay" data:

\section{Fitting our overall constant $\mathrm{K}$}

The effective Hamiltonian for $b \rightarrow s$ transitions is conventionally written as

$$
\mathcal{H}_{\mathrm{eff}}=-\frac{4 G_{F}}{\sqrt{2}} V_{t b} V_{t s}^{*} \frac{e^{2}}{16 \pi^{2}} \sum_{i}\left(C_{i} O_{i}+C_{i}^{\prime} O_{i}^{\prime}\right)+\text { h.c. }
$$

with the following set of dimension- 6 operators

$$
\begin{aligned}
O_{9} & =\left(\bar{s} \gamma_{\mu} P_{L} b\right)\left(\bar{\ell} \gamma^{\mu} \ell\right), & O_{9}^{\prime} & =\left(\bar{s} \gamma_{\mu} P_{R} b\right)\left(\bar{\ell} \gamma^{\mu} \ell\right), \\
O_{10} & =\left(\bar{s} \gamma_{\mu} P_{L} b\right)\left(\bar{\ell} \gamma^{\mu} \gamma_{5} \ell\right), & O_{10}^{\prime} & =\left(\bar{s} \gamma_{\mu} P_{R} b\right)\left(\bar{\ell} \gamma^{\mu} \gamma_{5} \ell\right) .
\end{aligned}
$$

In our model

$$
\begin{aligned}
& \mathcal{H}_{\text {eff }}=K V_{t b} V_{t s}\left(\frac{m_{\mu}}{m_{t}}\right)^{2} O_{9} \\
& \Longrightarrow K\left(\frac{m_{\mu}}{m_{t}}\right)^{2}=-\frac{4 G_{F}}{\sqrt{2}} \frac{e^{2}}{16 \pi^{2}} C_{9}=-\frac{G_{F}}{\sqrt{2}} \frac{\alpha}{\pi} C_{9}
\end{aligned}
$$


Fits to $R\left(K^{*}\right)$ and $R(K)$ assuming a single operator

New physics in $\mu$

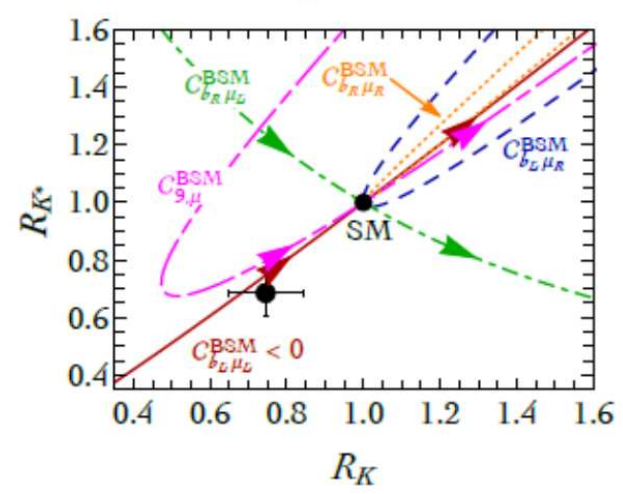

$C_{9}=-1.3 \pm 0.3$

Using

$$
\begin{aligned}
G_{F}^{0} & =1.1664 * 10^{-5} \mathrm{GeV}^{-2} \\
\alpha & =1 / 137.037 \ldots \\
m_{\mu} & =105.66 \mathrm{MeV} / \mathrm{c}^{2} \\
m_{t} & =173 \pm 0.8 \mathrm{GeV} / \mathrm{c}^{2}
\end{aligned}
$$

we get

$$
\begin{aligned}
K & \sim-\left(\frac{173}{0.106}\right)^{2} 1.1664 * 10^{5} \mathrm{GeV}^{-2} * \frac{-1.3}{\sqrt{2} \pi * 137.037} \\
& =\frac{1}{14 \mathrm{GeV}^{2}}
\end{aligned}
$$

\subsection{Fitting Our model for the $R(D), R\left(D^{*}\right)$, and $R(J / \Psi)$ anomaly in B-decay}

Since we do not have available any fitting of the coefficients to the dimension 6 operators for the $B \rightarrow D^{(*)} \tau \bar{v}_{\tau}$ process suspected to be the anomalous one ${ }^{1}$, we shall rather fit the $K$ value needed by comparing this process with heaviest lepton one from the " $R(K)$ and $R\left(K^{*}\right)$ " case just fitted above.

Under very crude simplifying assumptions, we indeed extract the ratio (averaged over the phase space) of the anomalous part for the decay $B \rightarrow D^{*} \tau \bar{v}_{\tau}$ relative to the anomalous part of the $B \rightarrow K^{*} \bar{\mu} \mu$ decay. We find that this ratio is very close to what we expect from our model. Indeed we find, that the ratio of the two anomaly-amplitudes is close to that of the ratio of the square of the masses of the involved (respectively heavier) leptons.

\footnotetext{
${ }^{1}$ We indeed take, in the cases of flavour universality violation, the anomalous process to be the one with the heavier one of the involved leptons in our non-perturbative model. Thus here the $\tau$ process.
} 


\section{$B \rightarrow D^{*} \tau \bar{\nu}_{\tau}, B \rightarrow K^{*} \mu^{+} \mu^{-}$Anomalous Amplitude Ratio}

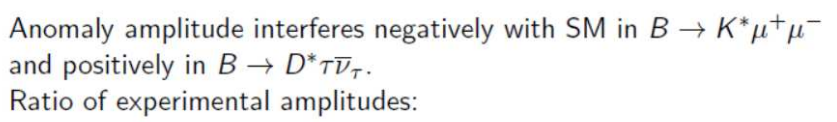

$\frac{A\left(B \rightarrow D^{*} \tau \bar{\nu}\right)_{\exp }}{A\left(B \rightarrow K^{*} \mu^{+} \mu^{-}\right)_{\exp }}=\sqrt{\frac{B R\left(B \rightarrow D^{*} \tau \bar{\nu}\right)}{B R\left(B \rightarrow K^{*} \mu^{+} \mu^{-}\right)}}=\sqrt{\frac{1.88 * 10^{-2}}{0.96 * 10^{-6}}}=140$

Deviations from SM:

$$
\frac{R(D *)_{\exp }}{R\left(D^{*}\right)_{S M}}-1=0.24 \quad \frac{R\left(K^{*}\right)_{\exp }}{R\left(K^{*}\right)_{S M}}-1=0.34
$$

$\Longrightarrow$ Ratio of anomalous amplitudes:

$$
\frac{A\left(B \rightarrow D^{*} \tau \bar{\nu}\right)_{\text {anom }}}{A\left(B \rightarrow K^{*} \mu^{+} \mu^{-}\right)_{\text {anom }}}=\frac{0.24 A\left(B \rightarrow D^{*} \tau \bar{\nu}\right)_{\exp }}{0.34 A\left(B \rightarrow K^{*} \mu^{+} \mu^{-}\right)_{\exp }}=\frac{0.24}{0.34} 140=99
$$

The crude assumptions we make for our very crude approximation comparing the two channels supposed to have an anomaly are:

- the amplitude is constant over all the phase space, so that it is as if the particles only went into one single state.

- The dominant anomalous contribution to the rate of the processes in question is the interference term between the Standard Model contribution and the anomalous part of the amplitude (it is the latter part that should be given by our estimate).

- It is supposed that there is no anomaly in the in the channels with the lighter one of the leptons. So the whole anomaly in the " $R\left(D^{*}\right.$ and $\left.R(J / \psi)\right)$ " case of deviation breaking universality between $\tau$ and $\mu$ or $e$ is blamed on the $\tau$-process. Similarly the whole deviation from lepton universality in the " $R\left(K^{(*)}\right)$ " case (actually between $\mu$ and $e$ ) is blamed on the muon channel. 


\section{Prediction of Anomalous Amplitude Ratio}

The one parameter $K$ in our model cancels out in the ratio of the anomalous amplitudes.

We thus predict:

$$
\begin{aligned}
& \frac{A\left(B \rightarrow D^{*} \tau \dot{\nu}\right)_{\text {arom }}}{A\left(B \rightarrow K^{*} \mu^{+} \mu^{-}\right)_{\text {anom }}}=\frac{V_{t b}^{2} V_{b c}\left(\frac{g_{2}}{g_{t}} \frac{g_{\tau}}{g_{t}}\right)^{2}}{V_{t b} V_{t s}\left(\frac{g_{u}}{g_{t}}\right)^{2}} \\
& =\frac{0.0412}{0.0404} \frac{g_{2}^{2}}{g_{t}^{2}} \frac{m_{\tau}^{2}}{m_{\mu}^{2}} \\
& =0.42+286 \\
& =120
\end{aligned}
$$

The experimentally estimated anomaly for the channel $B \rightarrow D^{*} \tau \bar{v}_{\tau}$ relative to the channel $B \rightarrow$ $K^{*} \mu^{+} \mu^{-}$is $99 / 120$ times our prediction, which is of course actually a remarkably good agreement. This means that, if we fitted our overall parameter $K$ to the reaction $B \rightarrow D^{*} \tau \bar{v}_{\tau}$, we would obtain a $K$ value 99/120 times the value we fitted above for the reaction $B \rightarrow K^{*} \mu^{+} \mu^{-}$, namely $K=$ $1 /\left(14 \mathrm{GeV}^{2}\right)$. Thus we can claim that the fit to the $R(D), R\left(D^{*}\right)$, and $R(J / \Psi)$ anomaly in B-decay gives $K=1 /\left(17 \mathrm{GeV}^{2}\right)$.

\subsection{Fitting to the muon magnetic moment anomaly}

The philosophy of our model for treatment of the nonperturbative effects is to look at the dimension 6 effective field theory coupling needed for providing the anomaly found experimentally, and seek to very crudely ${ }^{2}$ identify it with a dimension 6 operator which is very formally of the character of a product of two "currents" of dimension 3 each. Then the idea is to normalize these two currents by a close analogy to the normalization of the "currents" going into the normalizations of the single particle wave functions relativistically.

As stated here, we must admit that the rule does not sound extremely clear and definite. However we hope, by using the example of the anomalous part of the anomalous magnetic moment for the muon, to at least illustrate that there is a hope to suggest a clear rule - if not immediately then after a bit of experience:

Experimentally it has been found that the parameter $a_{\mu}=\frac{g_{\mu}-2}{2}$, "the anomalous magnetic moment for the muon", being half the difference of the g-factor for the muon magnetic moment from the free Dirac equation obtained value $g=2$, deviates by a small amount $\Delta a_{\mu}=a_{\mu}^{e x p}-a_{\mu}^{S M}=$ $2.68(63)(43) * 10^{-9}$ from the Standard Model prediction $a_{\mu}^{S M}=116591823 * 10^{-11}$. This is a priori to be explained, in the spirit of our story, by there being for some reason or another at first a high

\footnotetext{
${ }^{2}$ by changes that are at least no more than of order unity, but preferably just looking at analogies under Lorentz transformations
} 
dimension effective field theory term

$$
\Delta \mathscr{H}(x)=\Delta a * 2 \overline{\boldsymbol{\psi}}(x) \overrightarrow{\boldsymbol{B}}(x) \frac{e}{2 m_{\mu}} \overrightarrow{\boldsymbol{S}} \boldsymbol{\psi}(x) .
$$

Here the $\boldsymbol{\psi}(x)$ is the second quantized muon field and $\overrightarrow{\boldsymbol{B}}(x)$ is the second quantized magnetic field $\boldsymbol{B}_{1}=\boldsymbol{F}_{23} ; B_{2}=\boldsymbol{F}_{31} ; \boldsymbol{B}_{3}=\boldsymbol{F}_{12}$. Further $\vec{S}=\frac{1}{2} \vec{\sigma}$ is the muon spin matrix, and e.g. $S_{3}=\frac{1}{2} \gamma^{1} \gamma^{2}$. For non-relativistic particles without spin - just moving around with mass $m$ and charge $e$ - the ratio of the magnetic moment relative to the angular momentum is $\frac{e}{2 m}$. However when we have spin, this ratio may be different and the correction factor is called the $g$-factor and is here denoted by $g$.

Keeping track of the factors 2 we rewrite the extra effective term needed to provide the anomaly seen experimentally as

$$
\begin{aligned}
\Delta \mathscr{H}(x) & =\Delta a * 2 \overline{\boldsymbol{\psi}}(x) \overrightarrow{\boldsymbol{B}}(x) \frac{e}{2 m_{\mu}} \overrightarrow{\boldsymbol{S}} \boldsymbol{\psi}(x) \\
& =2 \Delta a * \overline{\boldsymbol{\Psi}}(x) \frac{1}{2} \boldsymbol{F}_{i j}(x) \frac{e}{2 m_{\mu}} \frac{1}{2} \gamma^{i} \gamma^{j} \boldsymbol{\psi}(x) \\
& =\frac{\Delta a}{2} * \frac{e}{2 m_{\mu}} \boldsymbol{F}_{i j}(x) \overline{\boldsymbol{\Psi}}(x) \boldsymbol{\gamma}^{i} \boldsymbol{\gamma}^{j} \boldsymbol{\psi}(x)
\end{aligned}
$$

The idea is that we should obtain such an effective term - or rather a quite covariant form of it - let us say for the effective Lagrangian density

$$
\Delta \mathscr{L}(x)=\Delta a * 2 \overline{\boldsymbol{\psi}}(x) \frac{1}{2} \boldsymbol{F}_{\rho v}(x) \frac{e}{2 m_{\mu}} \frac{1}{2} \gamma^{\rho} \boldsymbol{\gamma}^{v} \boldsymbol{\psi}(x),
$$

from a diagram of this type:

\section{Non-perturbative diagrams for $g-2$ of the muon}

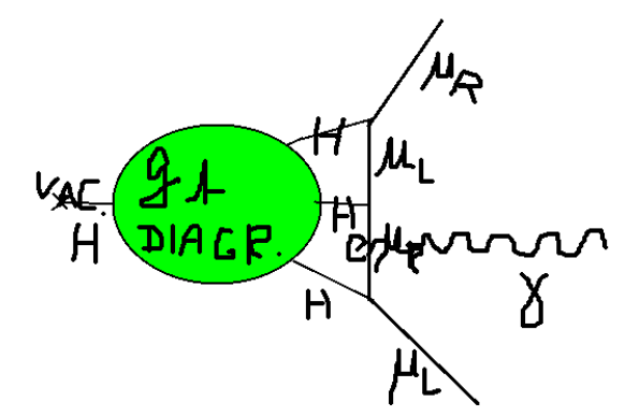

\footnotetext{
"Anomalous" anomalous muon magnetic mo-

ment nonperturbative contribution
}

We are led to this diagram since the anomalous magnetic moment coupling of the muon makes a left to right transition $\mu_{L} \rightarrow \mu_{R}$, requiring the external muon line to couple to an odd number (three) of Higgs lines. In order to conserve weak $S U(2)$ another Higgs line must emerge from the diagram and couple to the vacuum. 
This diagram has an external leg connecting to the vacuum Higgs field expectation value $<$ $\boldsymbol{\phi}_{H}>$, and corresponding to this fact the terms, we asked for above, are only of dimension 5, not of dimension 6 as we have announced we want to consider. Therefore we shall really replace our wanted term by an equivalent dimension 6 term

$$
\Delta \mathscr{L}(x)=\frac{\Delta a * 2}{<\boldsymbol{\phi}_{H}>} \boldsymbol{\phi}_{H} \overline{\boldsymbol{\psi}}(x) \frac{1}{2} \boldsymbol{F}_{\rho v}(x) \frac{e}{2 m_{\mu}} \frac{1}{2} \gamma^{\rho} \gamma^{v} \boldsymbol{\psi}(x) .
$$

The exercise now is to extract from this dimension 6 term some components, which can be written as a product of two "currents" of the type used to normalize wave functions in section (3.1), so as to obtain a normalization for the type of term we need for the anomaly in the muon anomalous magnetic moment.

To get matching with the special role of the index 0 in the normalization "currents", it may be easiest to work with the electric field, such as $\boldsymbol{E}_{3}=\boldsymbol{F}_{03}$ rather than with the magnetic field we truly set out to treat. However, because they are so closely related by Lorentz transformations, it is not so important as far as the magnitude is concerned; we can even keep control of the factors of 2 by such an analogy.

So let us take a Lorentz component after our favourite technical and pedagogical choice:

$$
\Delta \mathscr{L}(x) \text { "contains" } \frac{\Delta a * 2}{\left\langle\boldsymbol{\phi}_{H}\right\rangle} \boldsymbol{\phi}_{H} \overline{\boldsymbol{\psi}}(x) \boldsymbol{F}_{03}(x) \frac{e}{2 m_{\mu}} \frac{1}{2} \gamma^{0} \gamma^{3} \boldsymbol{\psi}(x) .
$$

Note that we removed the one factor $\frac{1}{2}$ because we now wrote explicitly indices 0 and 3 and thus do not sum over the permuted 3 and 0 .

In order to get it to match with the normalization "current", which we want, we have to make the approximation of replacing the $\gamma^{3}$, which at least has its square equal to unity, by unity itself.

We are looking to produce in our model the following type of term(s), because that is what seems to have been seen by the slight deviation of the very accurate muon magnetic moment anomaly calculation compared to experiment:

$$
\begin{aligned}
\Delta \mathscr{L}(x) \text { "contains" } & \frac{\Delta a * 2}{<\boldsymbol{\phi}_{H}>} \frac{e}{2 m_{\mu}} \boldsymbol{\phi}_{H}(x) \boldsymbol{F}_{03}(x) \overline{\boldsymbol{\psi}}(x) \frac{1}{2} \gamma^{0} \gamma^{3} \boldsymbol{\psi}(x) \\
\text { which taking } \boldsymbol{\gamma}^{3} \sim 1 & \text { gives } \\
\Delta \mathscr{L}(x) \text { "contains" } & \frac{\Delta a * 2}{<\boldsymbol{\phi}_{H}>} \frac{e}{2 m_{\mu}} \boldsymbol{\phi}_{H}(x) \partial_{0} \boldsymbol{A}_{3}(x) * \overline{\boldsymbol{\psi}}(x) \frac{1}{2} \gamma^{0} * 1 \boldsymbol{\psi}(x)
\end{aligned}
$$

where we have allowed ourselves to take a gauge $A_{0}=0$, so that the second term in $E_{3}$ was neglected.

Now we have come very close indeed to writing our wanted effective Lagrangian density term in the wanted way of being a product of two "currents" with their specific normalization attachable to the inner product normalization for wave functions. However the boson-"current"-factor has become mixed, in the sense that the two boson fields are from different particles: the Higgs and the photon respectively.

A little detailed problem is that the photon field, $A_{3}$ say, is "real" (or $\boldsymbol{A}_{3}$ hermitean), while the Higgs field $\phi_{H}$ is complex (or $\boldsymbol{\phi}(x)$ non-hermitean). This difference namely means that they should 
be normalized with "currents" deviating from each other formally by a factor 2 . Indeed, according to the rules discussed above in section 3.1, we should use:

$$
\begin{aligned}
& \text { "current" }\left.\right|_{\psi}=\bar{\psi}(x) \gamma^{0} \ldots \psi(X) \\
& \text { "current" }\left.\right|_{\text {photon }}=A_{i}(x) \partial_{0} A_{i}(x) \\
& \text { “current" }\left.\right|_{\text {Higgs }}=\phi^{*}(x) \stackrel{\leftrightarrow}{\partial_{0}} \phi(x),
\end{aligned}
$$

where one shall have in mind that $\overleftrightarrow{\partial_{0}}$ is modulo partial integration twice the single derivative $\partial_{0}$.

Let us further notice, that these "currents" are supposed to be a sum over contributions from all the polarization states for the fields being normalized: In the fermion case the summing over the Dirac basis is understood and in the Higgs case we imagine the contraction of the two component Higgs fields being understood, while in our photon analogy we have explicitly written an index $i$ supposed to be summed over, at least over as many photon components as are relevant.

For the case of mixing a real and a complex field, we already proposed above a compromise with a factor $\sqrt{2}$ replacing the either 1 or 2 in the respective real and complex case for constructing the "current". In fact our compromise means that we take the "mixed" "current" to be modulo "what we called partial integrations" (i.e. if we take it that we can change sign on a derivative and let it act to the opposite side without making any difference)

$$
\begin{aligned}
\text { "current }\left.\right|_{\text {Higgs,photon }} & =\sqrt{2} \phi^{*}(x) \partial_{0} A_{i}(x) \\
& \sim \frac{1}{\sqrt{2}} \phi^{*}(x) \overleftrightarrow{\partial_{0}} A_{3}(x) .
\end{aligned}
$$

(But now the summation index $i$ got a little bit too formal, in as far as we cannot contract it on the other side where we instead have the weak isospin doublet of Higgs components. So this compromise is indeed only very formal, but we use it anyway.)

Using this "mixed" "current", we can now write down our wished for effective Lagrangian piece - representing after being made covariant by extension especially also the magnetic moment interaction, which we truly need:

$$
\Delta \mathscr{L}(x) \text { “contains" } \frac{\Delta a * 2}{\left.<\boldsymbol{\phi}_{H}\right\rangle} \frac{e}{2 m_{\mu}} \frac{1}{\sqrt{2}} \text { “current" }\left.\right|_{\text {Higgs,photon }} \frac{1}{2} \text { “current" }\left.\right|_{\psi}
$$

But there is still a little problem: Above in (4.10) we had $\phi^{*} \partial_{0} A_{3}$, while the correct "current" $\left.\right|_{\text {Higgs photon }}$ has the summation over an $i$ meaning over the photon polarizations.

The identification possibilities as follows:

- We ignore the problem of whether we have only $A_{3}$ or the summation over i. Then:

$$
\begin{aligned}
\phi^{*} \partial_{0} A_{3} & \sim \frac{1}{\sqrt{2}} \text { "current" }\left.\right|_{\text {Higgs photon }} \\
& \sim \frac{1}{2} \phi^{*} \stackrel{\leftrightarrow}{\partial_{0}} A_{3}
\end{aligned}
$$


- We count say the occurrence of the order of 2 i-values summed over as a factor 2 say, and thus take

$$
\begin{aligned}
\phi^{*} \partial_{0} A_{3} & \left.\sim \frac{1}{2 \sqrt{2}}{ }^{\text {ccurrent }}\right|_{\text {Higgs photon }} \\
& \sim \frac{1}{2} \phi^{*} \stackrel{\leftrightarrow}{\partial_{0}} A_{3}
\end{aligned}
$$

According to our philosophy we should consider the operator factor "current" $\left.\right|_{\text {Higgs,photon }} *$ "current" $\left.\right|_{\psi}$ as normalized, so that our coefficient $K$ modified by what we call "suppression factors" should in our model be the coefficient of just this normalized term. Looking at the diagram we propose and noting that there are three non- $g_{t}$ couplings, which are muon Yukawa couplings and one electromagnetic coupling $e$, our $K$ with suppression factors becomes $K *\left(\frac{g_{\mu}}{g_{t}}\right)^{3} * e$. I.e. our model predicts an effective term of the appearance

$$
\begin{array}{r}
\left.K *\left(\frac{g_{\mu}}{g_{t}}\right)^{3} * e^{\text {“current }}\right|_{\text {Higgs,photon }} * \text { "current }\left.\right|_{\psi} \\
\text { to be identified with } \frac{\Delta a * 2}{<\phi_{H}>} \frac{e}{2 m_{\mu}} \frac{1}{\sqrt{2}} \text { “current" }\left.\right|_{\text {Higgs,photon }} \frac{1}{2} \text { “current" }\left.\right|_{\psi}
\end{array}
$$

This means that to get agreement for our model, the value of $K$ must be given by the equation

$$
K *\left(\frac{g_{\mu}}{g_{t}}\right)^{3} * e=\frac{\Delta a * 2}{<\phi_{H}>} \frac{e}{2 m_{\mu}} \frac{1}{2 \sqrt{2}}
$$

using the observed value of the anomaly $\Delta a=2.68 * 10^{-9}$. The derived $K$-value should of course be approximately equal to the $\mathrm{K}$-values obtained from the other anomalies.

The numerical evaluation for $K$ from this magnetic moment anomaly for the muon gives

$$
\begin{aligned}
K_{\text {from magn.mom. }}=K & =1637^{3} * \frac{2 * 2.68 * 10^{-9}}{246 \mathrm{GeV}} * \frac{1}{2 * 0.10566 \mathrm{GeV}} * \frac{1}{2 \sqrt{2}} \\
& =0.16 \mathrm{GeV}^{-2} \\
& =\frac{1}{6.25 \mathrm{GeV}^{2}}
\end{aligned}
$$

This calculation was made using (4.16), i.e. ignoring the summation, while if we instead use (4.18) and include a correction factor 2 for the summation, we get a fitted $K$-value which is a factor of 2 smaller, namely

$$
\begin{aligned}
K_{\text {from magn. mom.w. summing }} & =\frac{1}{2 * 6.25 G e V^{2}} \\
& =\frac{1}{12.5 G e V^{2}} .
\end{aligned}
$$

\subsection{Explaining Proton Radius Puzzle}

We now also want to explain the anomaly of the proton radius fitted to the muonic atomic physics spectrum seeming to be $4 \%$ smaller than the fit to the usual electronic atom spectrum in our model. The idea is of course that we shall propose some dimension 6 - like the other anomalies 
were explained by dimension $=6$ effective field theory terms - effective field theory term letting the muon interact with the hadronic matter in the proton providing a slightly different potential for the muon, while passing the proton. Such a correction to the potential for the muon inside the proton may easily be simulated by giving the proton a different radius. In fact the main effect of diminishing the radius of the proton on the electric potential felt by the muon - which is supposed to be the main interaction between the proton and the muon in usual electrodynamics - is to increase the potential a bit in the region filled by the proton. In our anomaly model the interaction of the muon is not just electromagnetic, as usually supposed, but partly due to an anomalous effective field theory term letting the muon interact with indeed gluon fields inside the proton.

The term, on which we shall settle as the one that can provide sufficiently strong interaction between the proton and the muon, is a term of the form

$$
\mathscr{L}_{e f f, \mu p}=K \frac{g_{S}^{2}}{g_{t}^{2}} * \frac{g_{\mu}}{g_{t}} * \boldsymbol{\phi}_{H}(x) \boldsymbol{A}_{\rho}^{a}(x) \boldsymbol{A}^{\rho a}(x) * \overline{\boldsymbol{\psi}}_{\mu} \ldots \boldsymbol{\psi}_{\mu}
$$

where $\boldsymbol{\psi}_{\mu}, \boldsymbol{A}_{\rho}^{a}$ and $\boldsymbol{\phi}_{H}$ are the fields for respectively the muon, the gluon and the Higgs particles. The coefficient in front of the field combination is a product of our fitting constant $K$, the ratio $\frac{g_{S}^{2}}{g_{t}^{2}}$ of the strong gauge coupling $g_{S}$ of QCD relative to the top quark Yukawa coupling $g_{t}$, and the ratio $g_{\mu} / g_{t}$ of the muon Yukawa coupling relative to the top yYukawa coupling. The dots "..." stand for some $\gamma$-matrices, but they are really just the unit matrix 1 in our present case. This proposal is actually very problematic in as far as it is not QCD gauge invariant. One must imagine that we extend it with further terms in some way to make it gauge invariant. To make it properly gauge invariant we may have to completely replace it by some quantity of a similar nature and dimension:

$$
A_{\rho}^{a} A^{\rho a} \rightarrow F_{\rho \sigma}^{a} F^{\rho \sigma a} / E_{\text {gluon }}^{2}
$$

In practice we shall be satisfied by estimating the average value of the $A A$ factor inside the proton, just by some dimensional argument, in terms of strong interaction scale parameters.

\subsection{Explaining our "theory"}

The principle of our model is that we imagine that we describe a very complicated diagram, in which by far the most copious vertex is the $g_{t}$ proportional vertex coupling the top quark or its doublet partner to the Higgs doublet. Then one associates the particles present in the effective field theory term we seek to obtain with the diagram, by slight modifications of such an otherwise only $g_{t}$ coupling diagram. Since we need a muon involved, we must at least extract one Higgs propagator and attach it to the muon by the Higgs muon Yukawa coupling $g_{\mu}$. This coupling couples a right chirality muon to a left chirality one. In addition we must have the gluon fields attached, by the gluons coupling to the color of some top quarks in the complicated diagram with only the $g_{t}$ coupling. This gives rise to the appearance of the extra factor $g_{S} / g_{t}$ one for each extracted gluon (field). By having two gluon fields extracted we can at least achieve gauge invariance under the global (part of the) gauge symmetry. Remembering that our diagram with only $g_{t}$ coupling and propagators taken to have no masses - and the propagators at all being basically ignored conserves the weak isospin $S U(2)$, we must have one more Higgs extracted, since otherwise the scalarly coupled muon would take off weak isospin. Therefore we had to have the extra field $\phi_{H}$ 
in the effective field theory term. We shall dispose of that by inserting for it its vacuum value $\left.<\boldsymbol{\phi}_{H}\right\rangle=246 \mathrm{GeV}$. Notice that we managed to make the term of dimension $=6$, as in the other anomalous terms we proposed.

With non-relativistic muons the Dirac field combination $\bar{\psi}_{\mu} \psi_{\mu}$ just becomes the numerical square of the non-relativistic muon field in the case of a single muon, and thus gives the muon (probability) density. Calculating perturbatively the important correction to the muon Schrødinger equation from our anomaly now becomes a correction to the potential $V_{\text {anomaly }}$ felt by the muon while being present inside the proton. This correction is given as

$$
V_{\text {anomaly }} \sim K \frac{g_{S}^{2}}{g_{t}^{2}} * \frac{g_{\mu}}{g_{t}} * \boldsymbol{\phi}_{H} \boldsymbol{A}_{\rho}^{a} \boldsymbol{A}^{\rho a}
$$

or rather we should estimate the average value of this operator inside the proton to obtain the potential it provides there.

The expectation value of $\boldsymbol{\phi}_{H}$ is of course easily taken to be $<\boldsymbol{\phi}_{H}>=246 \mathrm{GeV}$ as is well-known in vacuum.

From dimensional arguments the $\langle\boldsymbol{A A}\rangle$, although not meaningful for gauge reasons, may be taken to be an energy squared at the strong interaction scale such as $\Lambda_{Q C D}^{2}=(220 \mathrm{MeV})^{2}=$ $0.05 \mathrm{GeV}^{2}$.

We could also take the bag constant $B=10^{-3} \mathrm{GeV}^{4}$ from the MIT bag model and divide it by the needed square of a supposed energy of a gluon. Now it is possible to calculate the lowest energy level for a massless particle in a sphere of radius $R_{p}$ and that is found to be $\omega_{1} / R_{p}$ where $\omega_{1}=2.04$. Using $R_{p}=5 \mathrm{GeV}^{-1}$, we obtain $E_{\text {gluon }} \sim 2.04 / 5 \mathrm{GeV} \sim 0.4 \mathrm{GeV}$. In this way we estimate for a dimension $\left[\mathrm{GeV}^{2}\right]$ quantity extracted from QCD

$$
\frac{B}{E_{\text {gluon }}^{2}} \sim \frac{10^{-3} \mathrm{GeV}^{4}}{0.16 \mathrm{GeV}^{2}}=0.006 \mathrm{GeV}^{2}
$$

deviating by a factor 8 from the QCD-lambda estimate, but the QCD-lambda is rather arbitrary.

Then, taking $K \sim \frac{1}{15 \mathrm{GeV}^{2}}$, the anomalous potential felt by the muon inside the proton in our model becomes

$$
\begin{aligned}
V_{\text {anomaly }} & \sim K * \frac{g_{\mu}}{g_{t}} 246 \mathrm{GeV} * 0.006 \mathrm{GeV}^{2} \\
& \sim K * \frac{1}{1700} 1.5 \mathrm{GeV}^{3} \\
& \sim 6 * 10^{-5} \mathrm{GeV}
\end{aligned}
$$

\subsection{The potential observed $V_{\text {exp }, \mu}$}

Suppose we interpret the seemingly shrinking proton radius, when investigated by muonic atomic studies rather than by an ordinary electronic atom, as a change in the potential felt by the muon in the inside the proton. Then, if we treat the proton as a conducting object to evaluate the electric potential, the $4 \%$ decrease in radius in going from electron to muon means that the 
potential in the interior of the proton goes as follows:

$$
\begin{aligned}
\frac{\alpha}{R_{p}} \rightarrow \frac{\alpha}{R_{p}-4 \% * R_{p}} & \\
\text { meaning that } & \\
V_{\text {exp }, \mu}-V_{\text {from electron }}=\Delta V & \approx \quad+4 \% * \frac{\alpha}{R_{p}} \\
& =\quad 4 \% \frac{1}{137 * 5 \mathrm{GeV}^{-1}} \\
& =6 * 10^{-5} \mathrm{GeV}
\end{aligned}
$$

\subsection{Conclusion for Proton Radius Puzzle}

The accidentally perfect agreement for $K=\frac{1}{15 \mathrm{GeV}}$ means that we would fit $K$ to this value, if we use the proton radius puzzle anomaly to fit it.

If our picture is right - what our good agreement of course indicates - then the physically true radius of the proton is the one determined by the electronic atoms, for which there is a negligible anomaly. The physical effect, when one uses muons instead, is that there is an anomalous interaction between the muon and the hadronic matter in the proton. This interaction is attractive and functions as if the electric potential for the muon inside the proton material were deeper, but such an effect of a deeper potential for the negatively charged muon can be simulated by the electric potential inside the proton material being higher, an effect that could have been achieved by lowering the proton radius so that the positive charge of the proton were concentrated closer to the center of the proton. So it is a simulation effect and the true radius is the one gotten by means of the electron.

\subsection{Direct CP-violation $\varepsilon^{\prime}$}

In our analysis we assume that a $\Delta I=1 / 2$ rule applies to our non-perturbative $\mathrm{CP}$ violating $K \rightarrow 2 \pi$ decay amplitudes similar to that for the dominant CP conserving Standard Model amplitudes $\operatorname{Re} A_{0}$ and $R e A_{2}$. So, in the notation of Winstein and Wolfenstein [12], we take

$$
\frac{\operatorname{Im} A_{2}^{\text {anom }}}{\operatorname{Re} A_{2}} \sim \frac{\operatorname{Im} A_{0}^{\text {anom }}}{\operatorname{Re} A_{0}}
$$

Thus our non-perturbative $I=0$ and $I=2$ decay amplitudes should give similar contributions to $\varepsilon^{\prime}$. So, for simplicity, we just treat our non-perturbative $K \rightarrow 2 \pi$ as having isospin $I=0$ to estimate the value of $\varepsilon^{\prime}$. 


\section{Direct $\mathrm{CP}$ violation parameter $\epsilon^{\prime}$}

- $K^{0} \rightarrow \pi \pi$ described by $I=0$ and $I=2$ decay amplitudes $A_{I}$. $A_{0}$ dominates and $\mathrm{CP}$ violation generates small imaginary parts:

$$
\begin{aligned}
\epsilon^{\prime} & \sim \frac{1}{\sqrt{2}} \frac{\operatorname{Re} A_{2}}{\operatorname{Re} A_{0}} \frac{\operatorname{Im} A_{0}}{\operatorname{Re} A_{0}} \\
& \sim \frac{1}{30} \frac{\operatorname{Im} A_{0}}{\operatorname{Re} A_{0}}
\end{aligned}
$$

- $\operatorname{Re} A_{0}$ is given to leading order by $\mathrm{W}$ exchange with CKM mixing factors:

$$
\begin{aligned}
\operatorname{Re} A_{0} & =\frac{g_{2}^{2}}{M_{W}^{2}} V_{s u} V_{d u} \\
& =\frac{0.42}{(80.39)^{2} \mathrm{GeV}^{2}} * 0.225 \\
& =1.5 * 10^{-5} \mathrm{GeV}^{-2}
\end{aligned}
$$

\section{CP Violating Non-Perturbative Diagrams}

Quark Structure of decay process:

$$
K^{0}(s \bar{d}) \rightarrow \pi^{-}(d \bar{u}) \pi^{+}(\bar{d} u)
$$

- Non-perturbative diagrams have 4 external quarks with $s \rightarrow d$ and $\bar{d} \rightarrow \bar{d}$ transitions, giving CKM mixing factors $V_{t s} V_{t d}^{3}$.

- Contribution to anomalous CP violating amplitude:

$$
\begin{aligned}
\operatorname{Im} A_{0}^{\text {anom }} & =K V_{t s} V_{t d}^{3} \\
& =\frac{0.040 * 0.00867^{3}}{15 \mathrm{GeV}^{2}} \\
& =1.7 * 10^{-9} \mathrm{GeV}^{-2}
\end{aligned}
$$




\section{Evaluation of anomalous $\epsilon^{\prime}$ contribution}

The contribution of non-perturbative diagrams to direct CP violation is estimated to be:

$$
\begin{aligned}
\epsilon_{\text {anom }}^{\prime} & =\frac{1}{30} \frac{\operatorname{Im} A_{0}^{\text {anom }}}{\operatorname{Re} A_{0}} \\
& =\frac{1}{30} \frac{1.7 * 10^{-9} \mathrm{GeV}^{2}}{1.5 * 10^{-5} \mathrm{GeV}^{2}} \\
& =3.8 * 10^{-6}
\end{aligned}
$$

This is to be compared with the experimental value:

$$
\epsilon_{\text {exp }}^{\prime}=(3.6 \pm 0.5) * 10^{-6}
$$

again consistent with experiment

Remember that the Standard Model prediction for $\varepsilon^{\prime}$ is relatively small, and thus $\varepsilon_{\text {exp }}^{\prime}$ can roughly be identified with the anomaly - especially if the anomalous and Standard Model contributions have relative random phases. So that when we get a factor $\frac{3.8 * 10^{-6}}{3.6 * 10^{-6}}$ too big an anomaly, having used that $K$ were $\frac{1}{15 \mathrm{GeV}}$, it means that a fit of our model to $\varepsilon^{\prime}$ would give $K=\frac{1}{16 \mathrm{GeV}^{2}}$.

\section{Résumé Table}

The remarkable point of our whole investigation is that the different values of the overall scale parameter $K$ fitted to the different anomalies, indeed very different types of experiments, gets at least order of magnitudewise very much the same value from all the anomalies considered. This is seen in the following table:

\begin{tabular}{|c|c|}
\hline Observable & Fitted $K$ \\
\hline$B \rightarrow K^{*} \mu^{+} \mu^{-}$ & $\frac{1}{14} \mathrm{GeV}^{-2}$ \\
$B \rightarrow D^{*} \tau \nu_{\tau}$ & $\frac{1}{17} \mathrm{GeV}^{-2}$ \\
$a_{\mu}$ & $\frac{1}{12} \mathrm{GeV}^{-2}$ \\
$\varepsilon^{\prime}$ & $\frac{1}{16} \mathrm{GeV}^{-2}$ \\
Proton radius & $\frac{1}{15} \mathrm{GeV}^{-2}$ \\
\hline
\end{tabular}

Actually note that the deviations between the different fitted values of $K$ are only of the order of $20 \%$. But our calculations are typically so crude that agreement of the order of $20 \%$ must almost be considered only an accident. At least it would require some further argumentation to justify that we could do it with such an accuracy. Such argumentation could e.g. be saying that in the case of the two $B$-decay anomalies indeed a so small part of phase space plays the main role that a constant amplitude over the whole phase space would be justified. 


\section{Strong}

The thesis of the talk suggested from the $5-1=4$ ratios of anomaly strengths agreeing, because of of fitting 5 anomalies with the same $K$ value is: The Standard Model is perfectly O.K. even with Anomalies, provided one includes non-perturbative effects not only from Q.C.D., but also from a strong sector due to the Top Yukawa coupling $g_{t}$ being "strong".

Does this now mean: No New Physics ? Logically : Yes, no new physics! but in reality: The explanation for why $g_{t}$ is so strong? is suggested to be a new principle of ours, the so-called Multiple Point Principle [13]: Nature likes the couplings, such as e.g. $g_{t}$, to be critical, i.e. on a phase border (of some phase transition say)!

We and others have stated the "multiple point principle" in the form: the coupling constants get fine tuned so as to make several vacua have the same/or very small energy density.

In any case we know from the studies of supernovae etc. in astronomy, that the energy density (= cosmological constant) is very very small compared to any contribution to the energy density that could be expected from various sub-theories of the Standard Model, such as the strong interactions, or electrodynamics,.... Thus, from the particle physics point of view, the energy density of the vacuum is - in fact very mysteriously - extremely small. Thus postulating many vacua to exist, which have the same energy density would anyway imply that all the vacua have almost zero energy density. We thank Leonard Susskind for the proposal to formulate the multiple point principle as several vacua having $\sim$ zero energy density. By doing that you absorb the mysterious almost zero cosmological constant into the postulation of the multiple point principle.

\subsection{Analogy and Deviation from Q.C.D.}

Analogies: At the scales we can care for, the "weak" and "strong" scales, experimentally:

- Both

$$
\begin{aligned}
\sqrt{\frac{8+18 ?}{4 \pi}} g_{S}=\sqrt{(8+18 ?) \alpha_{S}} \approx 1 \\
\sqrt{\frac{16 ?}{4 \pi}} * g_{t} \approx 1,
\end{aligned}
$$

(see the significance of just this size below in subsection 6.2.)

- and they both run stronger towards the low energy scale $(t-->-\infty)$ and weaker towards the high energy scale $(t-->+\infty)$ (asymptotic freedom, almost for $g_{t}$ too):

$$
\frac{d g_{t}(t)}{d t}=\beta_{g_{t}}>0 \text { and } \frac{d \alpha_{S}(t)}{d t}=\beta_{\alpha_{S}}>0
$$

Difference between $g_{t}$ and $g_{S}$ :

The scale for $g_{t}$ being "strong" seems to be connected to the Higgs scale ??

But the Q.C.D. scale seems to be not connected with any Higgs-like field scale.

Our multiple point principle may give the explanation, that the Higgs field scale is adjusted to (fine tuned by our principle!) the scale of the strong scale $t$, where $g_{t}(t) \approx 1$, because the strong 
$g_{t}(t)$ produces a new phase "at the scale $t$ ". In fact we have previously estimated [14] the value of the top quark Yukawa coupling at the phase transition to be

$$
\left.g_{t}\right|_{\text {phase transition }}=1.00 \pm 0.14 \text {. }
$$

\subsection{How strong is strong?}

Except for $\alpha_{S}$, the strongest coupling in the Standard Model is the top quark Yukawa coupling $g_{t}$.

Let us look theoretically for how strong/large a coupling shall be to cause non-pertubative effects by Feynman diagrams with very many vertices and propagators becoming important, i.e. not becoming small.

We may crudely perform such an investigation by imagining going from one diagram - let us say already with many vertices and propagators - to one with one loop more. Adding one loop means, e.g. for Feynman diagrams like the ones with only $g_{t}$-vertices having three propagators attached, that one adds three propagators and two vertices. And then of course there shall be one more $d$-dimensional integral in the momentum representation, where $d$ is the dimension of spacetime. In the case of the $g_{t}$ diagrams with dimensionless coupling constants $\left[g_{t}\right]=1$, the extra integration will for large loop momenta get just equally many extra denominators as there are extra integrations in the $\frac{d^{d} q}{(2 \pi)^{d}}$ over the loop momentum $q$. In the large loop variable approximation we thus add, for each extra loop, a formally logarithmically divergent factor of the form

$$
g_{t}^{2} \int \frac{O(1)}{\left(q^{2}+\ldots\right)^{4 / 2}} \frac{d^{4} q}{(2 \pi)^{4}}
$$

for $d=4$. When this extra factor is found inside a convergent diagram or if the divergencies are taken care of somehow by renormalisation, we may not have to take the logarithmic divergence so seriously, but rather take it as roughly unity.

Adding one loop to a Feynman diagam:
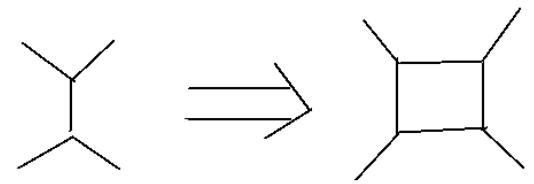

does it increase or decrease in numerical size ?

Very crudely a factor

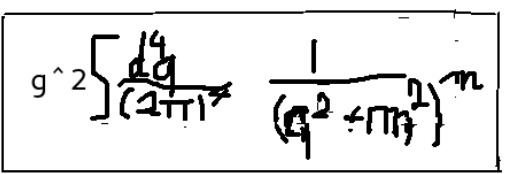

Now, in order to investigate what is the border line value between strong and weak coupling, we shall estimate when this extra factor is bigger or smaller than unity, because that signals whether the diagram of higher order - more loops - gets bigger or smaller than the foregoing one in the construction series. So the crudest estimate of the borderline coupling size is to require the coefficient to the logarithm in the logarithmic divergence appearing in (6.2) to be unity. 
The Borderline Coupling between Weak and Strong for Only One Component is $g \sim 4 \pi$. Taking very crudely by a "dimensional argument"

$$
\begin{aligned}
& \int \frac{d|q|}{|q|} \sim 1 \text { (by dimensional argument) } \\
& \text { and the borderline } \text { coupling } g_{\text {border }} \text { to } \\
& \text { have the extra factor by adding } \quad \text { a loop to satisfy } \\
& g^{2} \int \frac{d^{4} q}{(2 \pi)^{4}|q|^{4}} \approx 1 \text { (ignoring the mass squares }
\end{aligned}
$$

in the propagators)

So we get

$$
g_{\text {border }} \approx \sqrt{\frac{(2 \pi)^{4}}{\pi^{2}}}=4 \pi
$$

Rydberg constant $\approx$ Mass for a Coupling of "Order Unity"

$$
R_{\infty}=\frac{\alpha^{2} m_{\mathrm{e}} c}{4 \pi \hbar}=\frac{\alpha^{2}}{2 \lambda_{\mathrm{e}}}=\frac{\alpha}{4 \pi a_{0}}
$$

is of the order of the mass-energy $m_{\mathbf{e}} c^{2}$ for

$$
R_{\infty}=m_{\mathrm{e}} c^{2}
$$

implying

$$
\begin{aligned}
1 & =\frac{\alpha^{2}}{4 \pi c \hbar} \\
\text { or } & \\
\alpha^{2} & =4 \pi \text { for } c=\hbar=1 .
\end{aligned}
$$

meaning

$$
\begin{aligned}
e & =\sqrt[4]{(4 \pi)^{3}} \\
& =(4 \pi)^{3 / 4} \approx 6
\end{aligned}
$$

\section{Size of Coupling and Number of "Components"}

If there were e.g. a color quantum number taking $N$ values for the particle types encircling the loop, then there would be $N$ various loops for each one in case of no such inner degree of freedom. According to our philosophy of the extra factor by inserting a loop being of order unity

$$
g_{\text {border }}^{2} N \int \frac{d^{4} q}{(2 \pi)^{4}|q|^{4}} \approx 1
$$

then the $N$-dependence of the borderline coupling between the perturbative and non-perturbative regimes would be

$$
g_{\text {border }} \propto \sqrt{\frac{1}{N}} .
$$

For say 16 "Components" Borderline Coupling $\sim 1.5$ to 3 
Very crudely counting particle and antiparticle also as different "components" and counting together both the Higgs with its 4 real components and the top quark with its $3 * 2 * 2=12$ components, we get in total for the particles interacting via the top Yukawa coupling $g_{t}$ just $12+4=16$ components. Thus the borderline value for $g_{t}$ becomes

$$
g_{t \text { border }} \approx(6 \text { to } 4 \pi) / \sqrt{16}=1.5 \text { to } 3 .
$$

Experimentally

$$
g_{t} \exp =0.93_{5}
$$

\section{Neutral meson mixing, $K^{0} \bar{K}^{0}$ mixing.}

Our model for the anomalies, which seems so successful so far, has the problem that it predicts a far too big anomaly for e.g. $K^{0} \bar{K}^{0}$ mixing. Lattice calculations of this mixing and the mass difference of the two CP-eigenstates, or almost equivalently of the $K_{S}$ to $K_{L}$ mass difference, provide a Standard Model prediction [15] of

$$
\begin{aligned}
& \text { Bai (thesis): } \Delta M=(5.5 \pm 1.7) * 10^{-12} \mathrm{MeV} \\
& \text { Experiment: } \Delta M=(3.483 \pm 0.006) * 10^{-12} \mathrm{MeV}
\end{aligned}
$$

Although there would here be place for a negatively interfering anomaly of the same order of magnitude as the Standard Model prediction, there would of course not be place for a $3 * 10^{4}$ times larger anomaly than the Standard Model prediction. Taking our model straight away would give something of that order, so in this sense our simplest model is falsified. However such a relatively huge effect of our model pops up precisely in these mixing processes, because the amplitude or coefficient of the dimension 6 effective field term is only suppressed from our surprisingly large $K$ value by some mixing matrix element factors. The suppression is "only" by a factor $V_{t d} V_{t d}^{*} V_{t s} V_{t s}^{*}$ and thus the effective Lagrangian density term becomes of the order

$$
\begin{aligned}
& K * V_{t d} V_{t d}^{*} V_{t s} V_{t s}^{*} \overline{\boldsymbol{\psi}}_{s} \ldots \boldsymbol{\psi}_{d} * \overline{\boldsymbol{\psi}}_{s} \ldots \boldsymbol{\psi}_{d} \\
= & \frac{1}{15 \mathrm{GeV}^{2}} *|0.0404|^{2} *|0.00867|^{2} \overline{\boldsymbol{\psi}}_{s} \ldots \boldsymbol{\psi}_{d} * \overline{\boldsymbol{\psi}}_{s} \ldots \boldsymbol{\psi}_{d} \\
= & \frac{1}{15 \mathrm{GeV}^{2}} * 1.2 * 10^{-7} \overline{\boldsymbol{\psi}}_{s} \ldots \boldsymbol{\psi}_{d} * \overline{\boldsymbol{\psi}}_{s} \ldots \boldsymbol{\psi}_{d}
\end{aligned}
$$

while some of our other anomalies involve in addition Yukawa couplings for $\mu$ or $\tau$ leptons. Our dots "..." could typically be $\gamma_{\mu}$ and in fact it is likely to be accompanied with a $\gamma_{5}$ projection say.

Our effective field theory term here should be compared to the effective term estimated from the Standard Model by M. Gaillard and B. Lee [16], which gives the correct order of magnitude for the mass difference

$$
\mathscr{L}_{e f f}=-\frac{G_{F}}{\sqrt{2}} \frac{\alpha}{4 \pi} \varepsilon_{0} \cos ^{2} \theta_{C} \sin ^{2} \theta_{C} \overline{\boldsymbol{\psi}}_{s} \frac{1}{2}\left(1-\gamma_{5}\right) \boldsymbol{\psi}_{d} * \overline{\boldsymbol{\psi}}_{s} \frac{1}{2}\left(1-\gamma_{5}\right) \boldsymbol{\psi}_{d}
$$

where

$$
\begin{aligned}
\varepsilon_{0} & =\frac{\delta}{m_{W}^{2} \sin ^{2} \theta_{W}} \\
& =\frac{\delta}{(38 \mathrm{GeV})^{2}}=\frac{m_{c}^{2}}{(38 \mathrm{GeV})^{2}} .
\end{aligned}
$$


with a charm quark mass $m_{c}=1.3 \mathrm{GeV}$. So the ratio of our anomalous coefficient compared to the Standard Model coefficient, which fits experiment rather well, is

$$
\begin{aligned}
\frac{\text { "Our coefficient" }}{\text { "S.M. coefficient" }} & =\frac{\frac{1}{15 \mathrm{GeV}^{2}} * 1.2 * 10^{-7}}{-\frac{G_{F}}{\sqrt{2}} \frac{\alpha}{4 \pi} \varepsilon_{0} \cos ^{2} \theta_{C} * \sin ^{2} \theta_{C}} \\
& =\frac{8 * 10^{-9}}{m_{c}^{2} * 1.6 * 10^{-13} \mathrm{GeV}^{-2}} \\
& =5 /(1.3)^{2} * 10^{4}=3 * 10^{4} .
\end{aligned}
$$

Speculating that the typical energy scale at which our anomaly terms set in is of the order of $\mu=1 \mathrm{TeV}$ or the corresponding distance $0.2 \mathrm{am}\left(=0.2 * 10^{-18} \mathrm{~m}\right)$, our effective coupling of $8 * 10^{-9} \mathrm{GeV}^{-2}$ is in the units of this scale numerically

$$
\frac{8 * 10^{-9} \mathrm{GeV}^{-2}}{\mathrm{TeV}^{-2}}=10^{-2} .
$$

That would be o.k, but a priori one could have wondered if it would have been an unbelievably strong coupling.

However, if we think in terms of eigenstates of the conserved quantum numbers for our subtheory with only the $g_{t}$ coupling present, the particle to consider is say the dsb-quark linear superposition being in a weak doublet with the top quark. The whole such linear combination will not have these various mixing angle suppression factors and the effective field theory coefficient would rather be just $K$, so that we would wonder if the effective coupling in this language is possibly enormously strong. Again taking it that the scale is given by $\mu=1 \mathrm{TeV}$ would now have

$$
\frac{K}{T e V^{-2}} \sim 10^{4}
$$

and the coupling of these eigenstate particles would be unbelievably strong. Really it is presumably not possible to have couplings that are much bigger than unity in terms of the scale as unit.

In our model, wherein we have non-pertubative interactions, we may think of what happens at the micro-level as being the appearance of big bound states composed of many constituents. Then the absurdity of overly strong couplings would physically show up by the interacting particles hardly being able to penetrate into each other during the interaction.

Thus we shall now investigate in detail the possibility for the eigen-superpositions of quarks etc. penetrating into each other. We hope to argue that after all there will be difficulties in the particles penetrating into each other, and that thus the a priori effective coupling predicted in our model, relevant for the mixing of $K^{0} \bar{K}^{0}$ and forming the mass difference of the two superpositions $K_{L}$ and $K_{S}$, will turn out not to be so large in reality.

\section{Penetration Effect}

On a scale of length smaller than the scale $\mu \sim 1 \mathrm{TeV}$ at which the effective field theory is supposed to be used, we typically will have to think of the particles interacting in the effective field theory as being maybe even composite or at least consisting of a blob of surrounding constituents of virtual particles. In our scheme aiming at taking into account the effect of our "new strong 
interaction" of the particles interacting with the top-yukawa-coupling $g_{t}$, the important blob will mainly consist of three types of particles: the right top quark, the left top quark doublet and the Higgs, including the left dsb-quark as part of left top quark doublet.

When such blobs scatter in the way to be described by the effective field theory, it must then mean that these blobs are penetrating into each other while their constituents interact. If the interaction is sufficiently weak the blobs will, in first approximation, penetrate deeply through each other and the interaction may be thought of as a perturbative interaction between the constituents of the blob around one external particle with the blob around another external particle. However if the interactions are strong, one risks that one external particle together with its blob gets absorbed before even reaching very far into the other blob.

In principle this consideration gives us the opportunity to define a penetration depth for each species of external particle, telling how deep that sort of particle will penetrate before getting totally absorbed or transformed into a quite new particle.

In order to treat the calculation of $\varepsilon^{\prime}$ we shall find it useful to work with eigenstates of the CP-operator (in a certain frame).

\subsection{Eigenstates of penetration interaction}

In order to be able to describe relatively simply the absorption rate of various particles penetrating or attempting to penetrate into blob(s) around the other (external) particles, it is convenient to introduce some eigenstates of the absorption rate for these particles.

We should, so to speak, diagonalize the external particle species matrix of absorption or penetration degree.

In the first approximation we have our picture that the blob through which the particles with their blobs have to pass, consists of the particles/constituents, which interact via the Yukawa coupling $g_{t}$.

Naively one might therefore think of the medium, caused by the other particles than the one considered, could be taken as being quite independent of these other 3 particles; but this is for our treatment - especially our study of the the $\varepsilon^{\prime}$ CP-violating process - not a good idea. Rather we shall take it that the blob or medium, caused by the particles other than the considered particle, can be so to speak in two different states: one with $\mathrm{CP}=+$ and one with $\mathrm{CP}=-$. We shall then imagine that this CP-value for the medium is felt all over the medium. That is to say the particle passing through the medium will be absorbed in a way depending on this CP-eigenvalue, wherever in the medium it happens to get absorbed.

Thus, in order to specify the absorption rate for various particles, one has to have in mind that we have to discuss it for each CP-eigenvalue of the medium separately. Of course there is a very strong analogy between the two CP-eigenvalues for the medium: The absorption is strong whenever the quark-antiquark superposition (see discussion below) has the same CP-eigenvalue as the medium and weak when the eigenvalues of the medium and quark-antiquark superposition are different.

Much of the needed diagonalization is rather trivial to perform - at least in the approximation that we ignore the interaction with the Higgs vacuum expectation value and thus the different masses for the quarks and leptons - since it is only the specific linear combination of the three dsb-quarks, which form the weak doublet partner of the left chirality top-quark, that can interact 
via the $g_{t}$-coupling with the (eaten) Higgs bosons. In addition to these particles, it is only the top quark itself that interacts via the $g_{t}$-coupling. The rest of the quark linear combinations are in first approximation decoupled.

Further we have to care for approximate $\mathrm{CP}$ conservation. Therefore, in order to approximately diagonalize the strength of coupling to the $g_{t}$ coupling blobs, we shall choose CP-eigenstates. The quarks and the leptons in the usual basis do not diagonalize CP. We have to consider linear combinations of quarks and their anti-quarks! This might make the reader worried about superselection rules according to which one cannot make superpositions of states with different electric charges for instance. In our approximation of first only including the $g_{t}$ Yukawa coupling we have totally ignored the gauge fields, especially the electromagnetic fields, and thus the gauge symmetry is not so relevant in this scheme. The point is rather that, with the pure $g_{t}$ coupling, we have a theory with CP-invariance in first approximation. Thus, unless $\mathrm{CP}$ should get spontaneously broken, our first approximation model is totally CP-symmetric and mass - or here better interaction strength - eigenstates will be CP-eigenstates, unless they are degenerate. To be concrete, a priori there is mixing between a quark and the corresponding antiquark much like that between $K^{0}$ and its antiparticle $\bar{K}^{0}$, which is well-known. In the approximation of ignoring the CP-violation, it is well known that the eigenstates for the $K^{0}$-set are $K_{1}$ and $K_{2}$, which are CP-eigenstates. Of course one usually does not care so much for the corresponding eigenstates of CP among say the $d$ and the $\bar{d}$ quarks. This is because under normal conditions electric fields can too easily cause us to measure the charge and thus do not leave the $d$ and $\bar{d}$ system in peace to reveal its true eigenstates with $\mathrm{CP}$-eigenvalues. However, in the very short time their blobs interact only via the $g_{t}$ in our effective interactions, electromagnetism can be neglected and the true eigenstates can thus be of relevance.

Thus, in our approximation scheme, an important "eigen"-state for say the strength of the interaction are the CP-eigenstates in the subspace of top-right and its CP-transformed left-helicity anti-top, which we still say has right chirality. The same sort of CP-eigenstates can be formed from the antiparticle particle linear combination based on the left chirality top and similarly on the left chirality dsb-combination.

In order to make this a bit clearer, we list the eigenstates of this interaction strength in our approximation 


\section{The t-like quarks:}

"CP-odd right chirality top" $=\frac{1}{\sqrt{2}}\left(t_{R}-C P t_{R}\right)=\frac{1}{\sqrt{2}}\left(t_{R}-\bar{t}_{\text {left helicity }}\right)$

"CP-even right chirality top" $=\frac{1}{\sqrt{2}}\left(t_{R}+C P t_{R}\right)=\frac{1}{\sqrt{2}}\left(t_{R}+\bar{t}_{\text {left helicity }}\right)$

"CP-odd left chirality top" $=\frac{1}{\sqrt{2}}\left(t_{L}-C P t_{L}\right)=\frac{1}{\sqrt{2}}\left(t_{L}-\bar{t}_{\text {right helicity }}\right)$

"CP-even left chirality top" $=\frac{1}{\sqrt{2}}\left(t_{L}+C P t_{L}\right)=\frac{1}{\sqrt{2}}\left(t_{L}+\bar{t}_{\text {right helicity }}\right)$

"CP-odd dsb in doublet with $t_{L} "=\frac{1}{\sqrt{2}}\left(d s b_{L}-C P d s b_{L}\right)=\frac{1}{\sqrt{2}}\left(d s b_{L}-\bar{s} \bar{s} b_{\text {right helicity }}\right)$

"CP-even dsb in doublet with $t_{L} "=\frac{1}{\sqrt{2}}\left(d s b_{L}+C P d s b_{L}\right)=\frac{1}{\sqrt{2}}\left(d s b_{L}+\overline{d s} b_{\text {right helicity }}\right)$

Higgs-like

$$
\begin{aligned}
\text { CP-even neutral Higgs } & =\text { particle of } \operatorname{Re} \phi_{1} \\
\text { CP-odd neutral Higgs } & =\text { particle of } \operatorname{Im} \phi_{1} \\
\text { CP-even charged Higgs } & =\text { particle of } \operatorname{Re} \phi_{2} \\
\text { CP-odd charged Higgs } & =\text { particle of } \operatorname{Im} \phi_{2}
\end{aligned}
$$

In addition there are quark combinations, orthogonal to the here mentioned ones, which completely decouple from the $g_{t}$-material in the first approximation. Firstly all other 5 flavours of right chirality quarks (other than the top) are of this decoupled type, whether even or odd under CP. Secondly we can form linear combinations of the dsb quarks orthogonal to the combination in the SU(2)-doublet with the top and these left chirality combinations will also decouple. These particles will of course interact via the Higgses but with Yukawa couplings which are not competitive with $g_{t}$. Of course, in this very first approximation, the leptons are also decoupled.

So, in this approximation, particles which we here called decoupled will have infinitely long penetration depths. However the penetration depths of the even $\mathrm{CP}$ combinations mentioned above will be very short, in as far as they can go into the main diagram as replacements for one of the particles already participating in that diagram. In a medium with even CP-eigenvalue, the CP-odd combinations should couple so that the negative interference between the coupling of their two components would exclude their interaction and thus give them infinite penetration depths modulo CP-violation. However, the diagrams only involving the $g_{t}$ Yukawa coupling are by themselves $\mathrm{CP}$-conserving and thus, in the very first approximation, the CP-odd combinations decouple on the CP-even medium background. In an odd CP-eigenvalue medium, of course, it is opposite and the even CP particle has an infinite penetration depth, while the odd CP particle is absorbed very quickly.

Especially for the purpose of getting an understanding of how penetration goes in the case of CP-violating amplitudes, such as for calculating $\varepsilon^{\prime}$, we shall choose to describe what goes on by imagining that a quark superposition with its antiquark can only get absorbed. Then we shall consider scattering as being replaced by the absorbing medium somehow (later) emitting another particle thereby simulating a scattering. 
Let us in fact make a description of the scattering due to our effective field theory terms based on the following picture:

- Each external particle for our effective term interaction "sees" a blob/medium which is actually composed from the surrounding media of the other three external particles.

- This medium carries in a way extended over the whole medium the information as to whether it has a positive or a negative $\mathrm{CP}$ eigenvalue.

- We only consider the type of interaction that lets the particle focused on be absorbed by the medium of the other three. This means that, once the focused on particle has interacted/is absorbed by the medium it disappears and is no longer there. It can only be absorbed once.

- If the medium has the same CP-eigenvalue as the particle considered, then the absorption can be very strong and the particle will not reach far. If, however, the medium and the particle have opposite CP-eigenvalues, then the absorption as a quark and as an antiquark will interfere destructively and thus the absorption rate will be only proportional to CPviolation and thus be very small. Consequently, in the case of opposite CP-eigenvalues for the particle and the medium, the penetration depth can be large.

Among the cases we are interested in, it will be the scatterings or effective field interactions involving the $\mu$ and $\tau$ leptons that may penetrate through, while the left chirality quarks, especially the top quark itself, would seem liable to be absorbed already at the surface of the blob/medium. However, even these left-handed quarks can penetrate deeply if they have the opposite $\mathrm{CP}$-eigenvalue to that for the medium they pass through.

\subsection{Application of Penetration}

We now want to argue that a particle that is in an eigenstate of very strong absorbtion rate would only reach the surface of the blob or medium ball and thus have an appreciably less effective coupling in its effective interaction. Without such an effect our model for the coefficients of the various effective field theory terms would give a far too strong interaction term relevant for the mass splitting by mixing of the $K_{1}$ and $K_{2}$ in the $K^{0}-\bar{K}^{0}$ mixing. Our model for the coefficient to a term with 4, say, just $d$ and $s$ quarks without any CP-breaking would namely provide so large a coefficient that even alone it would give a much larger mass splitting than found experimentally. In other words our model would be severely falsified by e.g. $K^{0} \bar{K}^{0}$ mixing or by other meson mixings, if we do not modify the prediction for our anomaly in these mixing cases.

The idea now is that, for just these unwanted predictions, we can claim that the particles do not penetrate sufficiently deep for the rule(s) of our original model to become true. Indeed the quarks relevant for these mixings are supposed to interact by being interpreted as components of the very strongly interacting dsb-quark antiquark combination corresponding to the $S U(2)_{\text {weak }}$-partner of the top. It gets very quickly absorbed and we can very reasonably speculate that it only reaches the surface of the medium or blob discussed above.

So far the data would be well fitted by simply leaving out the contribution to mixing, in the case that none of the particles can penetrate because of such strong absorbtion. One has namely not seen any anomaly so far in the (CP-invariant) mixings. 
However these are the strongest terms according to our original rule and it does not seem reasonable to assume they should be totally removed. Rather we would say:

Once a particle hits the blob or medium it will either get absorbed sooner or later or it will escape on the opposite side. If it has a very strong absorption rate it will get absorbed almost immediately on the surface, while if it has a lower absorption rate it will penetrate deeper and for very low absorption rate easily escape out on the opposite side. But even the high rate of absorption particle gets absorbed and thus must have a higher or equally high chance of producing a process as the less strongly absorbed particles (in a given state of the medium w.r.t. CP eigenvalue). So there is a limit as to how strongly we can modify our model to reduce the effective field theory terms, which have all particles with strong absorption rate. We, so to speak, cannot make the coefficients smaller than an analogous process with a smaller absorption rate.

We will now discuss the important example of how much the "staying at the surface" effect can bring down our predictions for various specific processes:

Let us compare a CP-conserving effective field theory term with only $d$ and $s$ and $b$ quark or anti quark combinations/superpositions with a corresponding CP-violating process. Concretely you can think of the $\mathrm{CP}$-conserving process as giving the term leading to an anomalous contribution to the $K_{1}$ to $K_{2}$ mass difference, while the CP-violating term could be the term we use to explain the anomaly giving the anomalous $\varepsilon^{\prime}$ :

In the CP-conserving case the quark antiquark superpositions meet a medium with the same $\mathrm{CP}$-eigenvalue as themselves and thus they get absorbed extremely fast. In the CP-violating case the quark anti quark superposition meets a medium with the opposite CP-eigenstate as its own. Thus in the violating case the particle penetrates much further into the medium.

But now as a particle that has hit the medium region can only get absorbed once or escape on the other side, the chance of getting absorbed at all must be at least as big for the high rate of absorption particle as for the low rate of absorption one. In this example this means that the chance for the activity of the $\mathrm{CP}$-conserving process must be at least as big as that for the $\mathrm{CP}$-violating one.

Let us point out that by accident it could quite likely happen that say the CP-violating quark anti quark penetrating through the medium would be absorbed with high probability before reaching the other side. In such a case the total strength of the CP-violating and the CP-conserving processes would be equal to each other.

If this happens, we would in our now modified model obtain the prediction, that the anomalous part with and without CP-violation would be equally strong.

\subsection{Several CP-operators}

We have tended to talk loosely about the CP-operator as if there were a well-defined CPoperator at least in the rest frame of a particle. But the truth is that one can modify the CP-operator by multiplying it by some exponential of a linear combination of flavour charge operators. If for instance $C P$ is a CP-operator and the operators $N_{f}$ for various flavours $f$ are the number operators for the number of flavour $f$ quarks minus anti quarks, then we can define an infinity of other "CPoperators" by

$$
(C P)_{\text {new }}=C P \exp \left(i \delta_{f} N_{f}\right)
$$


Whenever you have a contribution to the Hamiltonian or a diagram with only two families, it is possible to choose the freedom in defining a $C P$ operator, i.e. the phases $\delta_{f}$ so that just this Hamiltonian contribution or diagram becomes CP-invariant under the $C P_{n e w}$.

If we think of the theory in our above used very crude approximation, in which we ignore masses in propagators because we ignore the vacuum Higgs field expectation value and rather consider the dsb-superposition connected by weak $S U(2)$ to the top as one particle, we have only involved one family, namely the third family. So for this case it is possible to choose a CP-operator that will be conserved in that approximation. For such a choice and such an approximation, the CP-odd superposition would not be absorbed at all in passing through a CP-even medium.

If we want to consider that there can be CP-violating effects and that such a CP-odd superposition will not penetrate infinitely deep into a $\mathrm{CP}$-even medium, then we can either:

- Choose a CP-definition that makes some other diagram than our anomalous one CP-invariant. In the case of our estimate above of $\varepsilon^{\prime}$ we have interference of our anomaly with a treediagram weak interaction term, involving of course the two lowest mass families when we think of the $K^{0}$ to $\pi \pi$ transition. With such a choice our anomaly diagram will no longer be CP-invariant.

- Alternatively we could start decorating our diagrams, which at first only have $g_{t}$ vertices diagrams, by allowing - in a more accurate approximation - also other smaller Yukawa couplings, or even transverse $W^{\prime}$ 's and $Z^{0}$ 's. With sufficiently many of these smaller couplings $\mathrm{CP}$-violation would at the end be unavoidable, in the sense that whatever way we would choose the definition of the $\mathrm{CP}$-operator at the end we could not achieve its conservation any more.

Using one of these formulations - either a foreign CP-choice or the inclusion of a next level of corrections - we can peacefully think of $\mathrm{CP}$ as slightly broken, and thus the CP-odd superposition going through the $\mathrm{CP}$-even medium would only get a finite penetration depth.

As an exercise we may estimate the strength of absorption of an odd-CP superposition going through an even $\mathrm{CP}$ medium in the two cases:

- The Wolfenstein representation [18] of the Cabibbo Kobayashi Maskawa matrix, with as real as achievable mixing elements $V$ 's, has only complex items in the third to first or opposite matrix elements. This corresponds to the fact that we can choose a CP-operator, which only gets broken when these third to first or opposite matrix elements are involved. So this Wolfenstein representation gives us a CP-choice of the type that keeps the tree-diagram dominating the $K^{0} \rightarrow \pi \pi$ process $\mathrm{CP}$-conserving. If we think of using this $\mathrm{CP}$-operator choice with our non-perturbative $g_{t}$ alone type of diagram, the coupling dsb-superposition (in doublet with top) will no longer behave in a CP-conserving way. This means that a linear combination of this superposition with its antiparticle superposition will not go totally unabsorbed through the even $\mathrm{CP}$ medium. The Wolfenstein mixing matrix between $\mathrm{t}$ and $\mathrm{d}$ has an order of unity phase but is numerically very small of the order of $1 \%$. Thus we can think of the odd-CP superposition of the dsb-quarks and anti-dsb quarks (in doublet with the top) as most likely being either ( $b$ or $\bar{b}$ ) or ( $s$ or $\bar{s}$ ) while there is only a probability of the 
order of one part in 10000 for it being a $d$ or $\bar{d}$. Only in the latter unlikely case can it violate the $\mathrm{CP}$ as suggested by the Wolfenstein formalism.

This thus suggests that, in this consideration, the odd CP eigenstate dsb combination interacting will penetrate about 10000 times further into the $\mathrm{CP}$-even medium than the corresponding even superposition. If you count that the $V_{t d}$ has about equal real and imaginary parts, we might say that the probability for absorption is even about a factor of 2 smaller. Thus the penetration should be about 20000 times deeper than that for the even CP partner.

Without violating the principle that the process amplitude for the even CP superposition cannot be lower than that for the odd one, we could at most postulate that the effect of the even $\mathrm{CP}$ superposition only reaching the surface could reduce its amplitude by a factor 20000 .

- Alternatively we could think of choosing at first a CP-definition leaving our first approximation "only $g_{t}$ diagrams" exactly CP-invariant, but then introduce smaller corrections by allowing also diagrams with a few smaller Yukawa couplings involving other families than the third one. Let us for simplicity choose to keep the approximation of no vacuum Higgs field and thus masslessness of all the involved quarks. It is indeed possible if we have in the perturbative corrections to our main diagrams in addition involved enough CKM-matrix elements to form a Jarlskog triangle, meaning a combination of the type

$$
J=\operatorname{Im}\left(V_{u s} V_{c b} V_{u b}^{*} V_{c s}^{*}\right)
$$

or any of the nine combinations of analogous flavours. By considering that these imaginary parts of products of CKM-matrix elements actually give the areas of the Jarlskog unitarity triangles, they have the same values. Thus, inserting into a diagram of ours, a set of non-maximal Yukawa couplings so as to deliver an expression leading to the $J$ makes the diagram develop an imaginary part, which cannot be removed by changing the phases built into the proposed $\mathrm{CP}$-operator with which a quark goes into the corresponding anti-quark - i.e. changing the $\delta_{f}$ 's above. That is to say such a decorated diagram will develop an imaginary part, meaning a breaking of $\mathrm{CP}$, for whatever definition of $\mathrm{CP}$ you might choose, being just $J$, the Jarlskog invariant. Most easily - with biggest value - we shall obtain the appearance of this kind of expression in the interference terms in the squared amplitude for the absorbtion of a CP-odd particle superposition passing the even CP medium say. Thus it will be absorbed by a rate proportional to this very Jarlskog invariant $J$. In the Wolfenstein representation its value is given by

$$
J \approx A^{2} \lambda^{6} \eta
$$

where $A=0.82$ and $\eta=0.34$ are of order unity while $\lambda=.22$ is the Cabibbo angle. Thus $J \approx 3.1 * 10^{-5}$. This is essentially the same as the factor $1 / 20000$ estimated above. So, in both cases, we conclude that the "only reaching the surface effect" could not suppress the $\mathrm{CP}$-even on CP-even medium absorption by more than this factor of $3.1 * 10^{-5}=\frac{1}{30000}$.

It seems we have learned the rule that, whatever the precise definition of the CP-operator considered, it will turn out that the absorption rate in the CP-violating case - when medium and particle 
superposition have opposite $\mathrm{CP}$ values - will be slower than the $\mathrm{CP}$-conserving rate by a factor being just equal to the Jarlskog invariant $J=3.1 * 10^{-5}$. So, measured in units of the penetration depth of the $\mathrm{CP}$-even particle into a $\mathrm{CP}$-even medium, the penetration depth of the CP-odd particle into the CP-even medium becomes the inverse of the Jarlskog invariant $J^{-1}$, thinking at first of an infinitely deep medium.

\subsection{Only-at-surface suppression.}

The reason for our interest in this penetration of especially the CP-eigenstates of quark type is that we want to estimate how the lack of penetration can modify the effective field theory coupling for a process - say a dimension $=6$ term - simulating our non-perturbative effects due to a strong coupling $g_{t}$. Instead of truly estimating the thickness of the medium we talk about and thereby the effect, we shall allow ourselves to introduce a second parameter to fit in our model. A priori we shall think of this second parameter to be related to the depth of the medium/blob relative to the depth into which the CP-nonviolating superposition - the even CP particles towards the even $\mathrm{CP}$ medium - penetrates with its very strong absorption. Let us denote the depth or diameter $D$ of the medium measured in units of the penetration depth of the even $\mathrm{CP}$ particle on an even $\mathrm{CP}$ medium. Then our modification of our original model consists in multiplying the effective field theory coefficient - which is what in the original model we calculate as our first parameter $K$ multiplied by some suppression factors - by an extra factor $\frac{1}{D^{\prime}}$. This extra factor takes care of the fact that a particle, which does not penetrate except into the surface, must have a smaller effective interaction strength than if it penetrates and can interact with the whole body of the medium.

- If the penetration depth $J^{-1}$ of a CP-odd superposition is longer than the diameter $D$, i.e. if $J^{-1}>D$, then the particle will often go through the medium and end up not being absorbed. In this case the probability for the absorption of the CP-odd particle can be small compared to its probability for hitting the medium at all. A corresponding CP-even particle will however, taking its penetration to be smaller than the diameter, always be absorbed. Thus the chance of absorption for the $\mathrm{CP}$-odd particle will be smaller than that for the $\mathrm{CP}$-even one. Consequently we also expect the effective coupling for the process involving the $\mathrm{CP}$-odd particle to be smaller than that involving the CP-even one instead. This means that the CP-even generated process, which is suppressed relative to our original model by a factor $D^{\prime}$, is actually suppressed by a factor $D$. The region through which the CP-odd particles can interact with the medium is namely $D$ times deeper than the region or depth for the CP-even particle. So indeed in this case of $J^{-1}>D$ :

$$
D^{\prime}=D
$$

- If on the other hand $J^{-1}<D$ the CP-odd particle gets absorbed inside the medium before reaching the other side. In this case the ratio of the penetration depths for the CP-odd to the CP-even particle (still in a CP-even background medium) is just the Jarlskog invariant inverted $J^{-1}$. That is to say we now have the extra suppression factor

$$
D^{\prime}=J^{-1} \text {. }
$$


Thinking of the difficult to estimate (second) parameter $D$ as a random variable, which we can only guess crudely if at all, we are actually interested in the distribution of the related parameter $D^{\prime}$, which is the only one going into our predictions. One would of course say that in such a statistical expectation there should be a finite non-zero probability for $D>J^{-1}$, so that even the odd-CP particle does not penetrate through the medium but gets absorbed on the way through. In this case we saw that $D^{\prime}=J^{-1}$. So this special value for the suppression factor $D^{\prime}=J^{-1}$ has a finite non-zero probability for occurring. So the probability distribution has a delta-function peak at this $J^{-1}$ value. If this is not the value for $D^{\prime}$ then we must have

$$
1 \leq D^{\prime} \leq J^{-1}
$$

It happens that our most simple prediction for the anomaly for the effective field theory term relevant for the $K^{0} \bar{K}^{0}$ mixing mass difference was just $3 * 10^{4}$ times larger than the whole Standard Model prediction for this term, a ratio accidentally(?) equal to the inverse of the Jarlskog invariant $J^{-1}=3 * 10^{4}$. Now, according to the above considerations, an especially likely value for the parameter $D^{\prime}$ which should correct a process or an effective field theory Lagrangian term is $D^{\prime}=$ $J^{-1}$. Taking this especially likely value $D^{\prime}=J^{-1}$, the simplest version of our model for the mixing term relevant for the mass difference would be cut down by a factor of just $D^{\prime}=J^{-1}=3 * 10^{4}$. This would make our corrected anomaly prediction be just of the same order as the Standard Model prediction in this case. Above in equations $(7.1,7.2)$ we saw that the theoretical prediction from the Standard Model for the mass difference was $5.5 * 10^{-12} \mathrm{MeV}$ while experiment gave $3.5 * 10^{-12}$ $\mathrm{MeV}$, i.e. theory was $\sim 50 \%$ too big.

So the deviation between theory and experiment in this mass difference case is really of the same order of magnitude as the theory prediction itself. So the best result for our model in the modified form would indeed be that there were an anomaly in this mass difference and we could, in this case, say that we predicted its order of magnitude correctly. However, it is of course o.k. just to say that our prediction for an anomaly in the modified version of our model just barely manages to be as small as the uncertainty. So our modified model has no problem with this process of mixing, although the simple model prediction looked like falsifying our model.

\section{A problem with $\varepsilon$}

At first it seems that, even after the inclusion of the story about the stopping at the surface of the too strongly interacting eigenstates of particles, our model still has the problem of predicting an anomaly in $\varepsilon$, the parameter of the first CP-violating experiment by Christensen, Cronin et al.

The problem is that, even including our suppression from particles that may not penetrate into the bulk of the interacting material, there is still an anomalous effect of our non-perturbative type present in the $K^{0}$ mixing system, making the transition between $K^{0}$ and $\bar{K}^{0}$. Before the suppression by the penetration effect of the $\mathrm{CP}$-conserving part, this $\mathrm{CP}$-conserving part would be dominant. However after this penetration effect suppression, we expect that the remaining anomaly could have a CP-violation effect of the same order as the CP-conserving one. Now we already found that the (CP-conserving) shift in the mass-difference due to our anomaly in fact turns out to be barely visible. This means that we actually predict that the present deviation of $\Delta M$ from the Standard 
Model prediction is already an anomaly of our type. But if so, then our about equally large CPviolating anomaly contributing to $\varepsilon$ would be dramatic, appreciably larger than the Standard Model CP-violation terms!

A priori it therefore looks that such a dramatic anomaly in the first discovered CP-violation parameter $\varepsilon$ would kill our model.

Here, however, we would like to point out that it is in reality not going to be quite as dramatic:

In fact we shall refer to a relation, derived under rather general (see e.g. [17]) conditions, concerning the possible values for the $\mathrm{CP}$-violation parameter $\varepsilon$ :

$$
\begin{aligned}
\frac{\left|\Gamma_{12}\right|}{\left|M_{12}\right|} * \sin (\phi) & =2\left(1-\frac{|q|}{|p|}\right) \\
& \approx 4 \operatorname{Re}(\varepsilon) .
\end{aligned}
$$

Here $\left|\Gamma_{12}\right|$ and $\left|M_{12}\right|$ are the numerical values of respectively the off-diagonal matrix element in the mass matrix for the $K^{0}$ to $\bar{K}^{0}$ system from the decay rate and from the mass. The angle $\phi$ is related to the argument of the ratio of the two types of off diagonal matrix elements $\Gamma_{12}$ and $M_{12}$,

$$
\phi=\arg \left(-\frac{M_{12}}{\Gamma_{12}}\right) .
$$

Calculating e.g. the important box-diagram amplitude from the Standard Model for the indirect CPviolation as well as for the mass difference, we see that the $\Gamma$ (and thus especially the off-diagonal $\Gamma_{12}$ ) represents the absorptive part, while the dispersive part is in $M$ having the off-diagonal $M_{12}$.

We can only obtain an absorptive part via the $K^{0}$ decay open channels and thus it only comes from diagrams involving such a low energy scale that energies of the order of the K-meson mass are achievable. The absorptive part from our anomalous term, for which the supposed mass scale is very high of the order of a half $\mathrm{TeV}$, will be totally negligible. Our anomaly will in practice only give a dispersive part and thus can only contribute to $M_{12}$ and completely negligibly to $\Gamma_{12}$.

In this light we see, that provided we have the relation (9.2) even when the anomaly is switched on, it is only $M_{12}$ but not $\Gamma_{12}$ that can be changed by the anomaly term. Thus the real part $\operatorname{Re}(\varepsilon)$ of the $\varepsilon$-parameter cannot get dramatically larger than the Standard Model value, since $\sin \phi$ can at most go up to unity, and the numerator $\left|\Gamma_{12}\right|$ cannot be changed by even a huge anomaly effect. Order of magnitudewise the anomaly prediction for $\operatorname{Re}(\varepsilon)$ is bounded from above by the Standard Model value.

Can we even get an effect of the anomaly for $\varepsilon$ downwards because of the anomaly effect in $\left|M_{12}\right|$ ?

It cannot be very dramatic, because the numerical value of the off-diagonal matrix element $\left|M_{12}\right|$ already contains the CP-conserving part, which gives rise to the mass difference $\Delta M$ between $K_{L}$ and $K_{S}$. So the relative anomaly content in $\left|M_{12}\right|$ should only be of the same order as the relative anomaly content in the mass difference. Although this mass difference could well be anomalous by a factor 2 say, there is no possibility for much more. But remember such a factor 2 was indeed what we a priori predicted.

So indeed our prediction for an anomaly in $\varepsilon$ is after all rather stabilized both against going up and going down. It is thus really not a killing of our model that the Standard Model calculation for the $\varepsilon$ - in our point of view happens to - agree(s) with experiment. 
We only have to suggest that the overall value of the anomaly fell a tiny bit to the low side in the $K^{0} \bar{K}^{0}$ mixing case, so that even the anomaly in the mass difference - which we may pretend to see - is a bit low. Thus the change in $\varepsilon$ by the anomaly gets to be of some order like say $30 \%$ and so is not really observable at present.

\subsection{Physics of our keeping anomaly in $\varepsilon$ down}

It is of course very crucial for rescuing our non-perturbative model for the anomalies that we understand how we avoided obtaining a huge anomaly for the CP-breaking parameter $\varepsilon$.

The physics behind this may be understood by thinking about the extreme case where one had a purely dispersive CP-breaking effect, coming from diagrams that could be completely represented by a dimension 6 effective field operator - like our model for the anomaly. The effective Hamiltonian for such a term would at first not have to have a real coefficient, in as far as it is a term adding say two strangeness units and then from hermiticity there should be a corresponding term removing two strangeness units. These two corresponding terms would of course have complex conjugate coefficients. But each of them can have a complex coefficient.

However now there is the freedom, discussed above in subsection 8.3, that one can construct several $C P$ operators, by supplementing one with exponentiated $i$ times flavour charge operators. When we consider a transition matrix element between a $K^{0}$ and a $\bar{K}^{0}$ state, such as $M_{12}$, then of course its phase can be changed by modifying the phase convention for the states with strangeness. Such a phase change is achieved for a CP-operator, if it is written in terms of $K_{1}$ and $K_{2}$, when this CP-operator is changed by being multiplied by a factor $\exp (i a S)$, where $S$ is the strangeness operator:

In fact, if we define the phases on the $\mid K^{0}>$ and $\mid \bar{K}^{0}>$ by defining them from

$$
\begin{aligned}
\mid K^{0}> & =\frac{1}{\sqrt{2}}\left(\left|K_{1}>+\right| K_{2}>\right) \\
\mid \bar{K}^{0}> & =\frac{1}{\sqrt{2}}\left(\left|K_{1}>-\right| K_{2}>\right) \\
\text { and } & \\
{ }^{\prime C} C P^{\prime \prime} \mid K_{1}> & =\mid K_{1}> \\
{ }^{\prime C} C P^{\prime \prime} \mid K_{2}> & =-\mid K_{2}>
\end{aligned}
$$

we could take

$$
\text { " } C P \text { " }=C P
$$

or we could take

$$
\text { “ } C P^{\prime \prime}=C P \exp (i a S) .
$$

In this way we can shift the relative phase of these states $\mid K^{0}>$ and $\mid \bar{K}^{0}>$ by adjusting the choice of the parameter $a$.

So we can now take such a definition of the $\mathrm{CP}$-operator " $C P^{\text {" }}$ that the transition matrix element $M_{12}$ becomes real. In this way we can make the purely dispersive matrix for the development of the $\mid K^{0}>$ plus $\mid \bar{K}^{0}>$ system " $C P^{\prime \prime}$-invariant. So in such a purely dispersive case - i.e. with no decay - there will be no violation of the chosen "CP" and thus there should be no decay of $K_{L}$, which will actually be $K_{2}$, into 2 pions. Thus indeed in such a case $\varepsilon=0$. 
This means that one can only achieve a non-zero $\varepsilon$ gradually as one screws up the absorptive part. But, as long as the absorptive part is in some sense small, $\varepsilon$ will be kept small in the same sense, even if there are huge dispersive terms.

Now if, as we suggest in our model, the anomaly only influences the dispersive part and that only by a relative magnitude close to being of order unity, but possibly a little less say of the order of $30 \%$, then the anomaly in $\varepsilon$ will also only be of this order. This is true even if it corresponds to a shift in the $M_{12}$ by about $30 \%$ and this anomalous shift has a quite different phase from the term it adds to, so that one would at first think it gave a huge $\mathrm{CP}$-violation.

\section{Conclusion}

We have looked at 5 "anomalies" meaning (small) deviations from the predictions of the Standard Model, and proposed that they are indeed due to non-perturbative effects rather than to genuine "new physics". We have fitted them order of magnitudewise by a somewhat arbitrary model based on the thought that, because of the rather large size of the top-Yukawa-coupling $g_{t}$, there are important Feynman diagrams of extremely/infinitely high order. We basically think of the extremely high order diagrams as just being modified by arranging them to have a few external lines. For practical purposes, in this philosophy, we then construct some effective field theory terms actually of dimension 6, which simulate the effect of the infinitely high order diagrams. Because we cannot evaluate the sum of the infinite order diagrams, we are forced to introduce one over-all scale parameter, which we call $K$.

Remarkably enough we obtain by the fitting of $K$ to the five different - and indeed very different in nature - anomalies order of magnitudewise the same value for $K$ ! This should be considered a great success of our idea, and is at least somewhat evidence for these anomalies indeed being due to non-perturbative effects.

Even more surprisingly the different fitted values only deviate from each other by up to $20 \%$, except for the anomaly in the muon magnetic moment. However, even for that case, we may by somewhat untrustable treatments of the factors of 2 obtain a value that could also agree within the $20 \%$. Strictly speaking there is possibly no reason that we should expect our model only to work order of magnitudewise. So we might hope in the future to convince ourselves and others that there is a way to make the treatment of the model such that one should expect a $20 \%$ accuracy. After all our procedure is only to modify Feynman diagrams, that a priori are (almost) the same for all the 5 considered anomalies.

\subsection{PREdictions}

Our model at first seemed to have a very severe problem:

It predicted far too large anomalies in $K^{0} \bar{K}^{0}$ mixing and other similar meson anti-meson mixings. With the purpose of avoiding such predictions disagreeing with experiment, we invented a physical mechanism of the penetration of too strongly coupling particles getting stopped, when in an interaction they are about to penetrate into each other (into the cloud of top quarks and Higgses around the other particle). But even this invented story only barely solves the problem, so we predict that we are very close to finding some anomalies in the mixing of meson systems. In fact the mass difference between $K_{L}$ and $K_{S}$ deviates from the Standard Model prediction by about a 
couple of standard deviations, and that would fit well with our estimate of an anomaly in this mass difference. We, so to speak, predict this should turn out to be a true anomaly.

For the $C P$-breaking $\varepsilon$ we at first seemed to get an intolerably large anomaly, completely dominating the Standard Model contribution. However, because our anomaly is completely dispersive and cannot change the absorptive part - $\Gamma_{12}$ the transition width - the anomaly in $\varepsilon$ cannot be so terribly large after all. It is, however, still a tiny bit of an accident that one has not seen any anomaly in $\varepsilon$ yet, but now it is not an outrageous accident.

A rather clean prediction from our model is that there shall be an even dominating anomaly in the decay channel analogous to the $R(K)$ and $R\left(K^{*}\right)$ case discussed but with $\tau$ replacing the $\mu$, i.e. in the channel:

$$
B \rightarrow K^{(*)} \tau^{+} \tau^{-}
$$

We namely get stronger and stronger anomalies the bigger the Higgs Yukawa coupling for the lepton.

\subsection{The inspiration, old ideas on bound state etc.}

The idea of non-perturbative effects due to the largeness of the top-Yukawa coupling $g_{t}$ can be considered as going back to our earlier work, where we speculated about the existence of bound states of 6 top +6 anti top quarks [14], caused by Higgs as well as gluon exchange. Such bound states are precisely the type of non-perturbative effects we could have in mind. But in the present work we took such an abstract view on the non-perturbative effects that they could have been many other configurations - new vacuum etc. - or just high order diagrams being important without any extra effects.

We estimated that the theoretical border for non-perturbative effects, taking into account the number of different components of Higgs-bosons and top-quarks interacting via the top-Yukawa coupling, is indeed very close to the experimental value of the top Yukawa coupling $g_{t}=0.935$. So indeed theoretically the coupling is on the borderline to be "strong".

Also our long discussed "Multiple point principle" [13] may have a place by explaining why the Yukawa coupling is just on the borderline of being strong (if indeed it is).

\subsection{Outlook}

Encouraged by the only $20 \%$ deviations between the different $K$-values fitted, it would be natural to attempt to redo our calculations fitting the $K$-parameter in such a way that such an accuracy would make sense.

A slightly related possible future project would be to estimate the sign of the effects. Indeed the signs vary from case to case, from anomaly to anomaly, in a way that would be hard even to give any meaning to, in the light of the different anomalies being so very different. But we think the first progress in estimating the sign could be to argue that the interaction between the muon and the hadronic matter in the shrunk proton puzzle has the sign corresponding to there being an attraction in the anomaly-term. This is very natural for a high mass scale effect (of a scalar shape, meaning no spin coming in in the supposed bound state). 


\section{Acknowledgement}

It should be mentioned that we have made appreciable progress on this work since the workshop in Corfu last year, where we basically only had 3 out of the now 5 anomalies under consideration.

One of us H.B. Nielsen wishes to thank the Niels Bohr Institute for being allowed to stay there as emeritus, and also for some economic support, although not sufficient for it all, as well as thanks to the conference organization, George Zoupanos et al.

Most of the progress was made while Colin Froggatt visited the Niels Bohr Institute and he thanks his friends - Chand and Wendy Sapru - for accommodation during this period. He also wishes to acknowledge hospitality and support from Glasgow University and the Niels Bohr Institute.

Also we wish to acknowledge an important question that was raised at the conference in Tallin in 2018 .

\section{References}

[1] LHCb collaboration, R. Aaij et al., "Test of lepton universality using $B^{+} \rightarrow K+\mu^{+}+\mu^{-}$decays", Phys. Rev. Lett. 113 (2014) 151601, [1406.6482].

LHCb collaboration, R. Aaij et al., "Angular analysis of the $B^{0} \rightarrow K^{0} \mu^{+} \mu^{-}-$decay using $3 f b^{-1}$ of integrated luminosity”, JHEP 02 (2016) 104, [1512.04442].

LHCb Collaboration, R. Aaij et al., "Test of lepton universality with $B^{0} \rightarrow K^{* 0} l^{+} l^{-}$decays" JHEP 08 (2017) 055, [1705.05802].

[2] "Beauty Quarks test lepton universality", cerncourier.com/2018/o3/23.

[3] LHCb collaboration, R. Aaij et al., "Measurement of the Ratio of Branching Fractions $" B\left(\bar{B}^{0} \rightarrow D^{*+} \tau^{-} \bar{v}_{\tau}\right) / B\left(\bar{B}^{0} \rightarrow D^{*+} \mu^{-} \bar{v}_{\mu}\right)$ ", Phys. Rev. Lett. 115 (2015) 111803.

Heavy Flavor Averaging Group (HFAG) collaboration, Y. Amhis et al., Averages of b-hadron, c-hadron, and $\tau$-lepton properties as of summer 2014, arXiv:1412.7515.

Belle collaboration, M. Huschle et al., "Measurement of the branching ratio of $B^{-} \rightarrow D^{(*)} \tau^{-} v_{\tau}$ relative to $B^{-} \rightarrow D^{(*)} \mu^{-} v_{\mu}$ decays with hadronic tagging at Belle", Phys. Rev. D92 (2015) 072014, [1507.03233].

Belle collaboration, A. Abdesselam et al., "Measurement of the branching ratio of $B^{0} \rightarrow D^{*+} \tau^{-} v_{\tau}$ relative to $B^{0} \rightarrow D^{*+} \mu^{-} v_{\mu}$ decays with a semileptonic tagging method, arXiv:1603.06711.

MILC collaboration, J. A. Bailey et al., $B \rightarrow D \mu v$ form factors at nonzero recoil and $|V c b|$ from 2+1-flavor lattice QCD, Phys. Rev. D92 (2015) 034506, [1503.07237].

BaBar collaboration, J. P. Lees et al., "Evidence for an excess of B decays $B \rightarrow D^{(*)} \tau^{-} v_{\tau}$ " Phys. Rev. Lett. 109 (2012) 101802, [1205.5442].

BaBar collaboration, J. P. Lees et al., "Measurement of an Excess of B Decays and Implications for Charged Higgs Bosons”, Phys. Rev. D88 (2013) 072012, [1303.0571].

[4] Thomas Teubner, “The anomalous anomaly” Nature Physics volume 14, page1148 (2018)

[5] R. Pohl, A. Antognini, F. Nez, et al. Nature 466, 213 (2010).

[6] A. Antognini, F. Nez, K. Schuhmann, et al. Science 339 , 417 (2013) 
[7] R. Pohl et al (CREMA Coll.) Science 353, 669 (2016)

[8] "Theoretical overview on the proton radius puzzle" - CERN Indico https://indico.cern.ch/event/658983/contributions/2915165

[9] A. Alavi-Harati et al. [KTeV Collaboration], "Measurements of direct CP violation, CPT symmetry, and other parameters in the neutral kaon system", Phys. Rev. D 67, 012005 (2003) Erratum: [Phys. Rev. D 70, 079904 (2004)] [hep-ex/0208007].

E. Abouzaid et al. [KTeV Collaboration], "Precise Measurements of Direct CP Violation, CPT Symmetry, and Other Parameters in the Neutral Kaon System", Phys. Rev. D 83, 092001 (2011) [arXiv:1011.0127 [hep-ex]].

C. Patrignani et al. [Particle Data Group], "Review of Particle Physics", Chin. Phys. C 40, no. 10, 100001 (2016).

A. J. Buras, M. Gorbahn, S. Jæger and M. Jamin, "Improved anatomy of...", JHEP 1511, 202 (2015) [arXiv:1507.06345 [hep-ph]].

T. Kitahara, U. Nierste and P. Tremper, "Singularity-free next-to-leading order $\Delta S=1$ renormalization group evolution and ..." JHEP 1612, 078 (2016) [arXiv:1607.06727 [hep-ph]].

[10] RBC and UKQCD Collaborations, Z.Bai et al., "Standard Model Prediction for Direct CP Violation in $K \rightarrow \pi \pi$ Decay", Phys. Rev. Lett. 115, 212001 (2015) [arXiv:1505.07863].

A. J. Buras and J. M. Gerard, "Upper bounds on $\varepsilon^{\prime} / \varepsilon$ parameters ... large N QCD and other news," JHEP 1512, 008 (2015) [arXiv:1507.06326 [hep-ph]].

A. J. Buras and J. M. Gerard, "Final state interactions in $K \rightarrow \pi \pi$ decays: $\Delta \mathrm{I}=1 / 2$ rule..." Eur. Phys. J. C 77, no. 1, 10 (2017) [arXiv:1603.05686 [hep-ph]].

[11] "On the anomalies in the latest LHCb data" - Hurth, T. et al -

CERN-TH-2016-046IPM-P.A-421MITP-16-003arXiv:1603.00865

"Flavour anomalies after the $R_{K *}$ measurement" G. D’Amico et al, JHEP 1709 (2017) 010 [arXiv:1704.05438]

[12] B. Winstein and L. Wolfenstein, "The search for direct CP violation", Rev. Mod. Phys. 65 (1993) 1113.

[13] D.L. Bennett, C.D. Froggatt and H.B. Nielsen, C94-07-20 (ICHEP 1994:0557-560), p.0557-560; Proc. 27th Int. Conf. on High Energy Physics, Glasgow, Scotland, 1994, eds. P. Bussey and I. Knowles (IOP Publishing Ltd., 1995), p. 557.

D.L. Bennett, C.D. Froggatt and H.B. Nielsen, in Perspectives in Particle Physics '94, eds. D. Klabucar, I. Picek and D. Tadic (World Scientific, Singapore, 1995), p. 255.

C.D. Froggatt and H.B. Nielsen, Phys. Lett. B368, 96 (1996).

[14] C. D. Froggatt and H. B. Nielsen "Remarkable coincidence for the top Yukawa coupling and an approximately massless bound state", Phys. Rev. D90 (2009) 034033 [arXiv:0811.2089].

[15] Z. Bai, "Neutral Kaon Mixing from Lattice QCD", Ph.D. thesis , Columbia University(2017).

Z. Bai, N. Christ and C. Sachrajda, "The $K_{L}-K_{S}$ Mass Difference", Lattice 2017, EPJ Web Conf. Vol. 175 (2018).

[16] M. K. Gaillard and B. W. Lee, Phys. Rev. D10 p. 897 (1974)

[17] Lectures on CP-violation, Nikhef, https://www.nikhef.nl/ h71/.../ppII-cpviolation-29012015.pdf

[18] L. Wolfenstein (1983). "Parametrization of the Kobayashi-Maskawa Matrix". Phys. Rev. Lett. 51 1945 (1983). 